\title{
MINIMAL POSITIVE HARMONIC FUNCTIONS
}

BY

\section{ROBERT S. MARTIN}

Introduction. One may ask how great generality in a domain is to be permitted if we are to have for this domain a formula possessing the more significant features of the Poisson-Stieltjes integral formula for the circle or the sphere $\left({ }^{1}\right)$. Even if there is agreement as to what the more important consequences of the formula are, there are two approaches, differing not so much in content as in emphasis, along which partial answers to the question lie. The first consists in determining hypotheses, as weak as possible, upon a domain under which all, or substantially all, of the important features of the formula admit of extension. The second consists in attempting to determine for each of the important consequences of the formula the class of domains for which it holds. While this sounds very much like the distinction between obtaining sufficient and obtaining necessary conditions for an extension of the formula together with all of its important features, actually it goes a little deeper, since the second viewpoint involves implicitly the notion that what a significant extension of the formula is may depend upon what it is going to be used for. It is a particular consideration from the viewpoint of the second approach which leads to the concept of a minimal positive harmonic function with which we are concerned in the present article.

A function positive and harmonic in a given domain we shall call minimal $\left({ }^{2}\right)$ - for this domain - if it dominates there no positive harmonic function except for its own constant submultiples. An important instance of this kind of function occurs in connection with the principle of Picard $\left({ }^{3}\right)$, whose relation

Presented to the Society, October 26, 1940; received by the editors May 10, 1940.

(1) For the two-dimensional case the possibilities have been rather fully discussed by G. C. Evans, The Logarithmic Potential, American Mathematical Society Colloquium Publications, vol. 6, New York, 1927, esp. chaps. 5 and 6. For the three-dimensional case under hypotheses related to bounded curvature of the boundaries, see C. de la Vallee Poussin, Propriétés des fonctions harmoniques dans un domaine ouvert limitê par des surfaces a courbure bornée, Annali della R. Scuola Normale Superiore di Pisa, (2), vol. 2 (1933), pp. 167-192; George A. Garrett, Necessary and sufficient conditions for potentials of single and double layers, American Journal of Mathematics, vol. 58 (1936), pp. 95-129. An approach from a different viewpoint is given by A. J. Maria and R. S. Martin, Representation of positive harmonic functions, Duke Mathematical Journal, vol. 2 (1936), pp. 517-529. A significant extension of the last results has recently been given by J. W. Green, Harmonic functions in domains with multiple boundary points, American Journal of Mathematics, vol. 61 (1939), pp. 609-632.

(2) It is scarcely necessary to mention that the present use of the term minimal bears no direct relationship to its use in connection with the problem of Plateau.

$\left({ }^{3}\right)$ See G. Bouligand, Fonctions Harmoniques, Principes de Dirichlet et de Picard, Mémorial des Sciences Mathématiques, no. 11, Paris, 1926; also, Etude des singularités de certains champs scalaires, Annales de l'École Normale Supérieure, (3), vol. 48 (1931), pp. 95-152. 
to a fairly general form of the integral formula has been discussed by Maria and the author $\left({ }^{4}\right)$. The general notion of a minimal function arises naturally when one considers from a more or less algebraic standpoint the way in which the integral formula represents the positive harmonic functions.

In space for a unit sphere with center at the point $O$, the formula in question is $\left({ }^{5}\right)$

$$
u(P)=\int_{\overline{O S}=1} F(S, P) d \mu\left(e_{S}\right) \quad(\overline{O P}<1),
$$

where $F(S, P)=\left(1-\overline{O P}^{2}\right) / \overline{S P}^{3}$, and where $\mu(e)$ is a finite, non-negative, completely additive function of Borel sets (mass distribution function) on the surface of the.sphere. The function $u(P)$ defined by this formula is always positive (non-negative) harmonic, and every such harmonic function is represented in this form by exactly one $\mu(e)$.

So far as the form of this representation is concerned, the two important features present are these: (1) A fixed family of functions, or basis, in terms of which the positive harmonic functions of the domain (sphere) are represented. This, of course, is the family of functions $F(S, P)$, where $S$ plays the part of a parameter or index. (2) A linear process, or rather, a positively homogeneous and additive family of such processes analogous to and having as instance the formation of finite linear combinations with positive coefficients of functions from the basis. This is realized as the process of integration with respect to the mass distribution. Because of the properties of the Stieltjes integral with respect to a "point mass" distribution, this family of linear processes must contain all "unit combinations" - that is, all combinations of the form of a positive multiple of a single element of the basis-and the unit combinations, and only those, must be non-expressible as the sum of two linearly independent combinations $\left({ }^{6}\right)$. In terms of these notions, there is a one-to-one representation of the positive harmonic functions of the domain as (generalized) positive linear combinations of functions from the basis.

Now the point to this formulation is that within the limitations described there is essentially only one basis that can be used in a representation of this type, namely, a suitably normalized family of minimal harmonic functions. More precisely, it can be shown that all functions of the basis must be positive harmonic and minimal in the domain and that exactly one positive multiple of each minimal function in the domain must occur in the basis. This is what the basis must be like, but it is, of course, not evident $a$ priori that in a given

(4) Maria and Martin, loc. cit.

(5) See H. E. Bray and G. C. Evans, A class of functions harmonic within the sphere, American Journal Mathematics, vol. 49 (1927), pp. 153-180.

${ }^{6}$ ) Without this restriction it would seem that formal resemblance to the Stieltjes integral is for the most part destroyed. The point need not be pressed, since it is relevant only to the motivation of the present developments and not to the developments themselves. 
domain minimal functions will exist, or, if they do, will exist in sufficient number to form the basis for such a representation. This leads to

PROBlem A. In a given domain, is the class of minimal functions sufficiently wide that, with a suitable normalization and a suitable definition of the linear process involved, it contains a basis for the positive harmonic functions of the domain?

A central result of the present article is to give a general affirmative answer to this question; that is, to show that the answer is in the affirmative for an arbitrary domain. It is also shown that the linear process can always be realized by an integral of the Stieltjes type, and further, that every positive harmonic function of the domain is the limit of functions which are finite linear combinations with positive coefficients of minimal functions. Thus, in so far as the properties outlined above are considered indispensable to what ought to be considered a significant extension of the Poisson-Stieltjes integral formula, the present analysis, in view of its general applicability, serves as a background against which to examine critically the possibility of obtaining extensions which preserve other important features of the integral formula.

The arguments employed do not involve in an essential manner the dimensionality of the Euclidean space in which the domain is supposed to lie. Thus though the results here are explicitly for the three-dimensional case, only obvious modifications would be necessary for the others. For unbounded domains in the plane, the exceptional behavior of the logarithmic potential at infinity necessitates minor changes in some of the statements.

We sketch briefly the scheme of the argument. Returning for a moment to the Poisson-Stieltjes integral formula, we recall that the function $F(S, P)$ occurring there is actually the normal derivative at the point $S$ of the Green's function $G(M, P)$ for the sphere. This normal derivative, not only in the case of the sphere but also in the case of any domain with a sufficiently smooth boundary, is equal (neglecting a positive factor independent of $P$ ) to the limit, as $M$ approaches $S$, of a quotient of the form $G(M, P) / G\left(M, P_{0}\right)$, in which $P_{0}$ has been chosen as some fixed point of the domain. Approach here is not restricted to be along the normal at $S$; in fact, the quotient may have a well defined limit for all modes of approach to a boundary point even though at that point there is no normal. In the case, however, of a sufficiently irregular domain, there will be boundary points at which the limit of the quotient is not determinate. This suggests the introduction of ideal boundary elements. Speaking roughly, we identify an ideal boundary element with the totality of modes of approach to the boundary for which the quotient has a specified limit. This procedure is carried out in $\$ 2$. With these ideal elements adjoined to the domain, we are able to obtain a convenient limiting form of the Riesz representation of superharmonic functions and, through it, an integral representation of positive harmonic functions bearing certain features of analogy 
with the Poisson-Stieltjes formula $(\$ 3)$. The feature which this representation lacks is uniqueness; there may be more than one distribution representing a given harmonic function. The failure of the uniqueness is shown in $\$ 4$ to be connected with the presence of non-minimal functions among the quotient limits. In the same section minimal functions are characterized, and it is shown that among all representations of a given harmonic function there is always exactly one (called canonical) which involves only minimal quotient limits. In this sense we recover the uniqueness of the representation and complete the answer to Problem A. In the concluding $\$ 5$ certain applications and examples are treated.

1. Auxilliary results on superharmonic functions. We begin by recalling a number of results concerning the solution of the generalized Dirichlet problem $\left({ }^{7}\right)$. Let $T$ be an open set in three-dimensional space; let $t$ be its boundary. If $P$ is a point of $T$, we denote by $m_{T}(e, P)=m(e, P)$ the mass distribution function resulting from sweeping a unit mass located at $P$ out of $T$. $P$ being fixed, $m(e, P)$ is a finite, non-negative, and completely additive function of sets $e$ measurable Borel. The total mass is located upon $t$, more precisely upon the boundary of that component (maximal open connected subset) of $T$ in which $P$ lies, and is, in case this component is bounded, equal to unity; if $P$ lies in an unbounded component of $T$, the total mass may be less. For fixed $e$, $m(e, P)$ is a non-negative harmonic function of $P$ in $T$.

Let $\phi(Q)$ be a function defined for $Q$ in $t$, measurable Borel, and summable over $t$ with respect to $m(e, P)$ for each $\left(^{8}\right) P$ in $T$. The integral

$$
u(P)=\int_{t} \phi(Q) d m\left(e_{Q}, P\right)
$$

taken in the sense of Radon-Stieltjes-Lebesgue, defines a harmonic $u(P)$ in $T$. We speak of this $u(P)$ as determined in $T$ by the boundary function $\phi(Q)$. In particular, if $\phi(Q)$ is continuous and, in case $t$ is unbounded, approaches zero at infinity, the function $u(P)$ is identical in each component of $T$ with the harmonic function determined there by the sequence solution, in the sense of Wiener, of the generalized Dirichlet problem for the boundary values $\phi(Q)$. Also under these circumstances $u(P)$ takes on continuously the boundary

(7) A rather complete bibliography of work relevant to this problem, together with an expository account, will be found in G. C. Evans, Dirichlet problems, American Mathematical Society Semicentennial Publications, vol. 2, New York, 1939, pp. 185-226. We shall cite: N. Wiener, Certain notions in potential theory, Journal of Mathematics and Physics (M.I.T.), vol. 3 (1924), pp. 127-146; C. de la Vallee Poussin, Extension de la méthode du balayage de Poincaré et problème de Dirichlet, Annales de l'Institut H. Poincaré, vol. 2 (1932), pp. 169-232; G. C. Evans, Potentials of positive mass, parts I and II, these Transactions, vol. 37 (1935), pp. 226-257, and vol. 38 (1935), pp. 201-236; O. Frostman, Potentiel d'Équilibre et Capacité des Ensembles, avec Quelques Applications a la Théorie des Fonctions, Thesis, Lund, 1935.

(8) Summability of the function for some one point $P$ in each component of $T$ is, of course, sufficient. 
values $\phi(Q)$ except possibly at the irregular points of $t$, and approaches zero at infinity. The irregular points of $t$ form at most a set of capacity $0\left({ }^{9}\right)$.

Now let $D$ be an arbitrary domain (open connected set) with boundary $d$. We shall assume in the sequel that $D$ is fixed. Certain results concerning the positive superharmonic functions in $D$ will be useful in the developments that follow.

Theorem I. Let $u(P)$ be positive and superharmonic in $D$. Let $\sigma$ be a subset of $D$ relatively closed in $D$. There exists uniquely a function $u_{\sigma}^{*}(P)$ defined in $D$ such that:

(a) $u_{\sigma}^{*}(P)$ is superharmonic in $D$.

(b) $u_{\sigma}^{*}(P)=u(P)$ at all points of $\sigma$ except possibly for those belonging to a subset of zero capacity.

(c) In $D-\sigma, u_{\sigma}^{*}(P)$ is identical with the function harmonic in $D-\sigma$ determined by the boundary function $\phi(Q)=\phi(\sigma, u ; Q)$, where

$$
\phi(\sigma, u ; Q)= \begin{cases}u(Q), & \text { when } Q \text { is a boundary point of } D-\sigma \text { lying in } D, \\ 0, & \text { when } Q \text { is a boundary point of } D-\sigma \text { ind. }\end{cases}
$$

It is convenient to introduce the following notation: if $f(P)$ is defined in $D$, non-negative and Borel measurable there, we denote by $f(\sigma ; P)$ the function defined as $f(P)$ for $P$ in $\sigma$, and for $P$ in $D-\sigma$ as the value at $P$ of the harmonic function determined in $D-\sigma$ by the boundary function $\phi(\sigma, f ; Q)$. It follows from this definition and the properties of an integral, in particular the integral of $(1.1)$, that $\left({ }^{10}\right)$ if $f_{n}(P) \uparrow f(P)$, or $f_{n}(P) \rightarrow f(P)$, then correspondingly we have $f_{n}(\sigma ; P) \uparrow f(\sigma ; P)$, or $f_{n}(\sigma ; P) \rightarrow f(\sigma ; P)$, provided only in the latter case that there is a summable majorant for all the boundary functions involved.

Assume for the moment that the theorem is true. Consider the function $u(\sigma ; P)$. This function and $u_{\sigma}^{*}(P)$, assumed to exist, agree except possibly on a subset of $\sigma$ of zero capacity, a fortiori almost everywhere in $D\left({ }^{11}\right)$. It follows that for $P$ in any bounded subdomain completely interior to (contained with its boundary in) $D$, and for all sufficiently large $n, A_{n} u(\sigma ; P)=A_{n} u_{\sigma}^{*}(P)$, where for an integrable $f(P)$ we denote by $A_{n} f(P)$ its integral mean (volume average) over a sphere of center $P$ and radius $1 / n$. Since $u_{\sigma}^{*}(P)$ is superharmonic ( $\left.{ }^{12}\right), A_{n} u_{\sigma}^{*}(P) \uparrow u_{\sigma}^{*}(P)$; hence $A_{n} u(\sigma ; P) \uparrow u_{\sigma}^{*}(P)$. Thus for a proof

( ${ }^{9}$ Capacity is used here in the sense of de la Vallée Poussin. In this sense, a set is of zero capacity if every distribution of positive mass which is positive on the set generates an unbounded potential.

(10) The symbols $\uparrow, \downarrow$, and $\rightarrow$ will denote respectively increasing, decreasing, and unspecified convergence of sequences of numbers, functions, or point-sets. Context will determine the sense.

(11) Sets of zero capacity are necessarily of zero spatial measure.

(12) For a connected exposition of various known properties of super- (sub-) harmonic functions the reader is referred to the tract by T. Rad6, Subharmonic Functions, Ergebnisse der Mathematik und ihrer Grenzgebiete, vol. 5, no. 1, Berlin, 1937. 
of the theorem we are led to investigate the existence and properties of the limit of the sequence $\left\{A_{n} u(\sigma ; P)\right\}$. Incidentally, the argument just given proves that if $u_{\sigma}^{*}(P)$ exists it is unique.

Suppose now that we have shown that (i) $u(\sigma ; P)$ is lower semicontinuous at all points of $D$ except possibly for a subset of $\sigma$ of capacity zero, and that (ii) $u(\sigma ; P)$ dominates its integral mean over any sphere of center $P$ contained with its boundary in $D$. If $D_{1}$ is a bounded domain completely interior to $D$, there follows from (ii) for all $P$ in $D_{1}$ and all sufficiently large $n$, $u(\sigma ; P) \geqq A_{n} u(\sigma ; P)$. For these $n$ and for all $P$ in a second domain $D_{2}$ completely interior to $D_{1}$, we then have for sufficiently large $m, A_{m} u(\sigma ; P)$ $\geqq A_{m} A_{n} u(\sigma ; P)=A_{n} A_{m} u(\sigma ; P)$. Thus $A_{m} u(\sigma ; P)$, since continuous, is superharmonic in $D_{2}$. From this it follows, in particular, that if $n<p$, then $A_{m} A_{n} u(\sigma ; P)=A_{n} A_{m} u(\sigma ; P) \leqq A_{p} A_{m} u(\sigma ; P)=A_{m} A_{p} u(\sigma ; P)$. On making $m \rightarrow \infty$ in the first and last members of this relation and using the continuity of the average functions, we obtain $A_{n} u(\sigma ; P) \leqq A_{p} u(\sigma ; P)(n<p)$. Since $D_{1}$ and $D_{2}$ are at our disposal, we have in $D, A_{n} u(\sigma ; P) \uparrow u^{\prime}(P) \leqq u(\sigma ; P)$, where $u^{\prime}(P)$ is superharmonic and where it is understood that approach of the prescribed type holds from some $n$ on in any bounded domain completely interior to $D$. Now since the lower limit of the sequence of its average functions clearly dominates a function in any of its points of lower semicontinuity, it follows from this last relation and (i) that $u(\sigma ; P)$ and $u^{\prime}(P)$ are identical except possibly on a subset of $\sigma$ of zero capacity. Thus $u^{\prime}(P)$ satisfies the requirements for $u_{\sigma}^{*}(P)$. To prove the theorem it therefore suffices to demonstrate (i) and (ii).

Now let $u(P)$ and $\sigma$ be as in the statement of the theorem. Let $f(P)$ be a function which is non-negative, continuous in $D+d$, zero on $d$, nowhere in $D$ greater than $u(P)$, and which approaches zero at infinity if $D$ is unbounded. Let $\Sigma^{\prime}$ be a bounded open subset of $\Sigma=D-\sigma$ completely interior to $D$ and having only regular boundary points. Write $\sigma^{\prime}=D-\Sigma^{\prime}$, and consider $f\left(\sigma^{\prime} ; P\right)$. From the definition of a superharmonic function follows $f\left(\sigma^{\prime} ; P\right) \leqq u(P)$. If $\Sigma^{\prime}$ runs through an increasing sequence whose sum is $\Sigma$, then $f\left(\sigma^{\prime} ; P\right) \rightarrow f(\sigma ; P)$. This is Wiener's result mentioned above. Thus we have $f(\sigma ; P) \leqq u(P)$. Now $u(P)$, being non-negative and lower semicontinuous in $D$, may (e.g. by defining it as zero on $d$ ) be extended so as to have these properties on the closed set $D+d$. It follows from this that $u(P)$ can be approximated in $D$ by an increasing sequence of functions satisfying the conditions imposed upon $f(P)$ above. Allowing $f(P)$ to run through such a sequence, we obtain $f(\sigma ; P) \uparrow u(\sigma ; P) \leqq u(P)$. At all points of $D$ except possibly those irregular boundary points of $\Sigma$ which are in $D$-and these form at most a subset of $\sigma$ of zero capacity-the functions $f(\sigma ; P)$ are continuous. Hence, with exactly the same possible exceptions, $u(\sigma ; P)$ is lower semicontinuous in $D$. This establishes (i), and proves, incidentally, that $u(\sigma ; P) \leqq u(P)$.

To prove (ii), it is convenient to write $v(P)=u(\sigma ; P)$. Let $\Sigma^{\prime \prime}$ be any 
bounded open set completely interior to $D$ having only regular boundary points; write $\sigma^{\prime \prime}=D-\Sigma^{\prime \prime}$. We prove that $v(P) \geqq v\left(\sigma^{\prime \prime} ; P\right)$. Assume that $f(P)$ is continuous in $D$, non-negative, and not greater than $v(P)$. From the definitions and $v(P) \leqq u(P)$, it follows that the difference $w(P)=v(P)-f\left(\sigma^{\prime \prime} ; P\right)$ is non-negative in $\sigma+\sigma^{\prime \prime}$. In the remaining points of $D$, those of the open set $\Sigma \cdot \Sigma^{\prime \prime}$, the function $w(P)$ is harmonic and bounded from below. Since a boundary point of $\Sigma \cdot \Sigma^{\prime \prime}$ is in $\sigma+\sigma^{\prime \prime}, w(P)$ has a non-negative lower limit in anty such boundary point which is also a point of lower semicontinuity of $v(P)$. As this means all boundary points except possibly those of a set of zero capacity, $w(P)$ is also non-negative in $\Sigma \cdot \Sigma^{\prime \prime}\left({ }^{13}\right)$; that is, $v(P) \geqq f\left(\sigma^{\prime \prime} ; P\right)$. Now $v(P)$, though not necessarily lower semicontinuous, could be made so by modifying it on a set of zero capacity $\left({ }^{14}\right)$. Thus we can find an increasing sequence of continuous functions approaching $v(P)$ except on a set of zero capacity. On allowing $f(P)$ to run through such a sequence and observing that sets of zero capacity are null sets with respect to the swept-out mass in (1.1), we obtain for $P$ in $\Sigma^{\prime \prime}, f\left(\sigma^{\prime \prime} ; P\right) \uparrow v\left(\sigma^{\prime \prime} ; P\right)$. This proves that $v(P) \geqq v\left(\sigma^{\prime \prime} ; P\right)$, since the equality holds by definition in $\sigma^{\prime \prime}$.

Now let $P_{1}$ be any point of $D$; let $S_{\rho}$ be the open sphere of center $P_{1}$ and radius $\rho$, where $\rho$ is less than distance $\left(P_{1}, d\right)$. Denote by $s_{\rho}$ the boundary of $S_{\rho}$. If we take $\Sigma^{\prime \prime}$ above as $S_{\rho}$, the function $v\left(\sigma^{\prime \prime} ; P\right)=v\left(D-S_{\rho} ; P\right)$ is given for $P$ in $S_{\rho}$ by Poisson's integral with the boundary function $v(Q)$ on $s_{\rho}$. In particular, for $P=P_{1}$

$$
v\left(P_{1}\right) \geqq v\left(D-S_{\rho} ; P_{1}\right)=\frac{1}{4 \pi \rho^{2}} \int_{\delta_{\rho}} v(Q) d Q,
$$

where integration is with respect to area. On multiplying the first and last members of this relation by $4 \pi \rho^{2}$ and integrating with respect to $\rho$ between the limits 0 and $r$, where $r$ is less than distance $\left(P_{1}, d\right)$, we obtain

$$
\frac{4}{3} \pi r^{3} \cdot v\left(P_{1}\right) \geqq \int_{S_{r}} v(P) d P,
$$

integration now being with respect to volume. This establishes (ii), and completes the proof of Theorem I.

In the next theorem are listed a number of useful elementary properties of the function $u_{\sigma}^{*}(P)$ defined above. In the statement of the theorem, $u(P)$,

$\left.{ }^{(13}\right)$ If not, then for some negative number the set of boundary points at which the lower limit of the function does not exceed this number is of positive capacity. Cf. O. D. Kellogg, Foundations of Potential Theory, Berlin, 1929, p. 335. It may be noted that Kellogg uses the term capacity in the sense of Wiener, but for closed sets this coincides with the present usage.

(14) For example, this could be done by redefining the function as its lower limit at those points where it fails to be lower semicontinuous. These points form at most a set of zero capacity. Since the lower limit is not decreased in any point by this process, the modified function must be lower semicontinuous. 
$v(P)$, etc. will be understood to denote non-negative superharmonic functions in $D ; \sigma, \tau$, etc., will be subsets relatively closed in $D$.

THEOREM II.

(a) $u(P) \geqq u_{\sigma}^{*}(P) \geqq 0$, for all $P$ in $D$.

(b) If $u(P) \geqq v(P)$ at all points of $\sigma$ except for a subset of zero capacity, then $u_{\sigma}^{*}(P) \geqq v_{\sigma}^{*}(P)$ for all $P$ in $D$.

(c) $(u+v)_{\sigma}^{*}(P)=u_{\sigma}^{*}(P)+v_{\sigma}^{*}(P)$.

(d) $(c \cdot u)_{\sigma}^{*}(P)=c \cdot u_{\sigma}^{*}(P), c$ being a non-negative number.

(e) If $u_{n}(P) \rightarrow u(P)$ at all points of $\sigma$ except for a subset of zero capacity, and if there exists a majorant $U(P)$ to the $u_{n}(P)$, where $U(P)$ is superharmonic in $D$, then $\left(u_{n}\right)_{\sigma}^{*}(P) \rightarrow u_{\sigma}^{*}(P)$ in $D$, except for a subset of $\sigma$ of zero capacity. Lacking the majorant $U(P)$, we may still assert that $\lim _{\inf _{n \rightarrow \infty}}\left(u_{n}\right)_{\sigma}^{*}(P) \geqq u_{\sigma}^{*}(P)$ at all points of $D-\sigma$.

(f) If $\sigma \leqq \tau$, then $\left(u_{\tau}^{*}\right)_{\sigma}^{*}(P)=\left(u_{\sigma}^{*}\right)_{\tau}^{*}(P)=u_{\sigma}^{*}(P)$.

(g) If $\sigma \leqq \tau$, then $u_{\sigma}^{*}(P) \leqq u_{\tau}^{*}(P)$. More generally, if $\sigma_{n} \uparrow \sigma$, then $u_{\sigma_{n}}^{*}(P)$ $\uparrow u_{\sigma}^{*}(\dot{P})$.

(h) $u_{\sigma+\tau}^{*}(P) \leqq u_{\sigma}^{*}(P)+u_{\tau}^{*}(P)$.

It is pertinent to make the obvious remark that if $u(P)$ and $v(P)$ are superharmonic, and $u(P) \geqq v(P)$ at almost all points of $D$, then consideration of volume averages extends the inequality at once to all points of $D$. Thus (a) holds, since it was established almost everywhere in the proof of Theorem I. The statements (b), (c), and (d) are easy consequences of the definitions and the remark just made.

For (e), a point $P$ of $\sigma$ where $\left(u_{n}\right)_{\sigma}^{*}(P) \rightarrow u_{\sigma}^{*}(P)$ fails must be a point where $u_{n}(P) \rightarrow u(P)$ fails, where $u_{\sigma}^{*}(P) \neq u(P)$, or where, for some $n,\left(u_{n}\right)_{\sigma}^{*}(P) \neq u_{n}(P)$. All such points form at most a subset of $\sigma$ of zero capacity. In $D-\sigma$, in case the majorant $U(P)$, exists, the boundary functions $\phi\left(\sigma, u_{n} ; Q\right)$ are dominated by the summable function $\phi(\sigma, U ; Q)$, and the result is a consequence of Lebesgue's convergence theorem. If $U(P)$ fails to exist, we may still apply a well known lemma of Fatou.

Turning to $(f)$, we observe that when $\sigma \subseteq \tau, u_{\tau}^{*}(P)$ and $u(P)$ agree at all points of $\sigma$ except those of a set of zero capacity. From (b) then follows $\left(u_{\tau}^{*}\right)_{\sigma}^{*}(P)=u_{\sigma}^{*}(P)$. Using this result in conjunction with (a) and (b), we obtain

$$
\left(u_{\sigma}^{*}\right)_{\tau}^{*}(P) \geqq\left(\left(u_{\sigma}^{*}\right)_{\tau}^{*}\right)_{\sigma}^{*}(P)=\left(u_{\sigma}^{*}\right)_{\sigma}^{*}(P)=u_{\sigma}^{*}(P) \geqq\left(u_{\sigma}^{*}\right)_{\tau}^{*}(P),
$$

which proves the other half of (f).

The first statement of (g) follows from (f) and (a). For the more general statement, write $v_{n}(P)=u_{\sigma_{n}}^{*}(P)$. Then $v_{n}(P) \uparrow v(P)$, where $v(P)$ is superharmonic and not greater than $u(P)$. In any point of $\sigma$ where, for some $n$, $v_{n}(P)=u(P)$ holds, thus in all points of $\sigma$ except for a set of zero capacity, $v(P)=u(P)$. From this and (e) it follows that $\left(v_{n}\right)_{\sigma}^{*}(P) \rightarrow u_{\sigma}^{*}(P)$ in $D$ except 
for a subset of $\sigma$ of capacity zero. From (f), however, $\left(v_{n}\right)_{\sigma}^{*}(P)=v_{n}(P)$. This implies (with the aid of the remark at the outset of the proof) that $u_{\sigma}^{*}(P)$ $=v(P)$ in $D$. Thus we have $v_{n}(P) \uparrow u_{\sigma}^{*}(P)$.

For $(\mathrm{h})$, write $v(P)=u_{\sigma}^{*}(P)+u_{\tau}^{*}(P)$. It is clear from the non-negative character of the functions and from the definitions that $u(P) \leqq v(P)$ at all points of $\sigma+\tau$ except for a set of zero capacity. Thus using (b), (c), and (f) in succession, we have

$$
\begin{aligned}
u_{(\sigma+\tau)}^{*}(P) & \leqq v_{(\sigma+\tau)}^{*}(P)=\left(u_{\sigma}^{*}\right)_{(\sigma+\tau)}^{*}(P)+\left(u_{\tau}^{*}\right)_{(\sigma+\tau)}^{*}(P) \\
& =u_{\sigma}^{*}(P)+u_{\tau}^{*}(P) .
\end{aligned}
$$

We conclude the present section by obtaining for a special case the Riesz representation of the function $u_{\sigma}^{*}(P)$. It may be recalled that the generalized Green's function $G(M, P)$ for $D$ is defined as $1 / \overline{M P}$ minus the harmonic function $v_{M}(P)$ determined in $D$ by the boundary function $\phi(Q)=1 / \overline{M Q}$. $G(M, P)$, thus defined for $M$ and $P$ in $D$, is non-negative and symmetric in its arguments. For fixed $M$ it is harmonic in $P$ except at $P=M$ and approaches zero at every regular point of $d$; it also approaches zero at infinity if $D$ is unbounded.

THEOREM III. If $u(P)$ is non-negative, superharmonic, and continuous in $D$, and if $\sigma$ is a bounded closed subset of $D$, then $\left.{ }^{15}\right)$

$$
u_{\sigma}^{*}(P)=\int_{\sigma} G(M, P) d \nu_{\sigma}\left(e_{M}\right),
$$

where $\nu_{\sigma}(e)$ is a finite, non-negative and completely additive function of Borel sets having its total mass in $\sigma$.

It is convenient to take Riesz's result in a form due to Frostman $\left({ }^{16}\right)$, who proved that the functional

$$
J(\nu)=\frac{1}{2} \int_{\sigma} \int_{\sigma} G(M, P) d \nu\left(e_{M}\right) d \nu\left(e_{P}\right)-\int_{\sigma} u(P) d \nu\left(e_{P}\right),
$$

under the same hypotheses upon $u(P)$ and $\sigma$ as in the statement of the present theorem, is minimized by a unique $\nu(e)=\nu_{\sigma}(e)$ among all non-negative mass distributions $\nu(e)$ whose total mass is in $\sigma$. The function $v(P)$ given by

$$
v(P)=\int_{\sigma} G(M, P) d \nu_{\sigma}\left(e_{M}\right)
$$

(15) The hypotheses here are obviously unnecessarily restrictive, but the result in its present form is adequate for our purposes.

${ }^{\left({ }^{16}\right)}$ O. Frostman, La méthode de variation de Gauss et les fonctions sousharmoniques, Acta Litterarum ac Scientiarum Regiae Universitatis Hungaricae Francisco-Iosephinae, Szeged, vol. 8 (1936-1937), pp. 116-126. 
is equal to $u(P)$ at all points of $\sigma$ except possibly for those of a subset of capacity zero, and nowhere in $D$ exceeds $u(P)$. The proof of the present theorem reduces to showing that $v(P)=u_{\sigma}^{*}(P)$.

The function $v(P)$ is superharmonic in $D$, harmonic in $D-\sigma$ and approaches zero at every regular point of $d$ and also at infinity in case $D$ is unbounded. From the inequality $v(P) \leqq u(P)$, the continuity of $u(P)$, and the lower semicontinuity of $v(P)$, follows the continuity of $v(P)$ in any point where $v(P)=u(P)$. Thus in $D-\sigma$ the function $v(P)$ approaches boundary values $\phi(\sigma, u ; Q)$ at all boundary points of $D-\sigma$ except possibly for a set of zero capacity. The function $u_{\sigma}^{*}(P)$ satisfies exactly the same boundary conditions in $D-\sigma$, and, since both functions are bounded, they are identical there. In $\sigma$ the functions differ on at most a set of zero capacity; hence they are identical in $D$.

2. The ideal boundary elements and the metric $\rho$. In this section we define for $D$ a set of ideal boundary elements and derive certain properties of the domain with these elements adjoined.

Let $P_{0}$ be a point of $D$ chosen arbitrarily but fixed for the ensuing discus$\operatorname{sion}\left({ }^{17}\right)$. We denote by $G(M, P)$ the generalized Green's function for $D$, and define for $M$ and $P$ in $D$

$$
K(M, P)= \begin{cases}G(M, P) / G\left(M, P_{0}\right) \quad\left(M \neq P_{0}\right) \\ 0 & \left(M=P_{0} ; P \neq P_{0}\right), \\ 1 & \left(M=P=P_{0}\right) .\end{cases}
$$

The function $K(M, P)$ is for fixed $M$ a non-negative harmonic function of $P$ except at $P=M$; its value at $P=P_{0}$ is 1 . For fixed $P, K(M, P)$ is continuous as a function of $M$ except at $M=P$.

Consider now a sequence $\left\{M_{n}\right\}$ of points of $D$ having no point of accumulation in $D$. In any bounded closed subdomain of $D$, the functions $K\left(M_{n}, P\right)$ form, from some $n$ on, a bounded sequence of harmonic functions of $P$-thus a normal family $\left({ }^{18}\right)$. A subsequence of these functions, therefore, is convergent in $D$ to a positive harmonic function. A sequence $\left\{M_{n}\right\}$ of points of $D$ having no accumulation point in $D$, for which the corresponding $K\left(M_{n}, P\right)$ have the property of the subsequence just mentioned-that is, converge to a harmonic function in $D$-will be called fundamental. We have just seen that any se-

(17) As will be seen presently, the particular choice of this point makes no essential difference in the structure of the ideal boundary or in the theory that follows. In fact, the only effect of a change in the choice is to multiply the functions $K(M, P)$ by a factor $1 / K\left(M, P_{0}^{\prime}\right)$, where $P_{0}^{\prime}$ is the new normalizing point.

(18) The normalization at $P_{0}$ entails the boundedness both from above and positively from below of the function $K(M, P)$ for $P$ in a bounded domain containing $P_{0}$ uniformly in $M$ when $M$ is kept more than a fixed positive distance from this domain. Kellogg, loc. cit., pp. 263-265. The normal family property is a well known consequence of Harnack's inequality and the theorem of Ascoli. 
quence of points of $D$ without accumulation point in $D$ has a fundamental subsequence. Two fundamental sequences are called equivalent if their corresponding $K(M, P)$ 's have the same limit. This has the usual properties of an equivalence relation.

DEFINITION 1. The class of all fundamental sequences equivalent to a given one determines (or, simply, is) an ideal boundary element of $D$. The set of all ideal boundary elements of $D$ will be denoted by $\Delta$, and the set $D+\Delta$, by $\mathcal{D}$.

The domain of definition of $K(M, P)$ may now be extended by writing

$$
K(M, P)=\lim _{n \rightarrow \infty} K\left(M_{n}, P\right) \quad(M \text { in } \Delta ; P \text { in } D),
$$

where $\left\{M_{n}\right\}$ is any fundamental sequence determining $M$. For $M$ in $\Delta$, $K(M, P)$ is thus a positive harmonic function of $P$ in $D$ having the value 1 for $P=P_{0}$.

Evidently the function $K(M, P)$ is characteristic of the point $M$ in the sense that the identity of two points of $\mathcal{D}$ is equivalent to the equality of their corresponding $K(M, P)$ 's as functions of $P$. Thus, in view of the possible application of the normal family theory, it is to be expected that $\mathcal{D}$ can be given a topology with respect to which it is compact and with respect to which $K(M, P)$ as a function of $M$ possesses certain continuity properties. This may be shown more explicitly by introducing a metric. Actually, the precise analytic form of the metric we choose to introduce is not of great importance in the present developments but has some technical advantages.

Definition 2. Select a fixed sphere $\Sigma$ completely interior to $D$ having, say, $P_{0}$ for center. For $M$ and $M^{\prime}$ in $\mathcal{D}$ we define $\left({ }^{19}\right)$

$$
\rho\left(M, M^{\prime}\right)=\int_{\Sigma} \frac{\left|K(M, P)-K\left(M^{\prime}, P\right)\right|}{1+\left|K(M, P)-K\left(M^{\prime}, P\right)\right|} d P,
$$

in which integration is with respect to volume and in which the integrand is defined conventionally if $P=M$ or $M^{\prime}$.

THEOREM I. The function $\rho\left(M, M^{\prime}\right)$ is a metric in $\mathcal{D}$. With respect to it $\mathcal{D}$ is complete and compact, $D$ is open, and $\Delta$ is the boundary of $D$. The relative topology in $D$ arising from the metric is equivalent to the original topology there.

That $\rho\left(M, M^{\prime}\right)$ is finite, non-negative, symmetric, and that it satisfies the triangular inequality and vanishes if $M=M^{\prime}$ is clear from (2.1). If $\rho\left(M, M^{\prime}\right)=0$, the integrand in (2.1), since non-negative, must vanish in every point of $\Sigma$ at which it is continuous. Hence, $K(M, P)=K\left(M^{\prime}, P\right)$ for all $P$ in $\Sigma$ except possibly $P=M$ or $P=M^{\prime}$. Harmonic continuation extends

(19) Taking the integrand in this form is, of course, a purely technical device for obtaining a bounded integrand. Cf. S. Banach, Théorie des Opérations Linéaires, Warsaw, 1932, pp. 9-10. 
the equality to all $P$ in $D$. Thus $\rho\left(M, M^{\prime}\right)=0$ implies $M=M^{\prime}$. This establishes the first statement of the theorem.

The remainder of the proof will be carried out in a number of brief steps. A sequence $\left\{M_{n}\right\}$ of points of $\mathcal{D}$ convergent to a point $M$ in the sense of the metric $\rho$ will be called $\rho$-convergent to $M$.

(i) A sequence $\left\{M_{n}\right\}$ of points of $D$ convergent to a point $M$ of $D$ is $\rho$-convergent to $M$. For, if in (2.1) $M$ is taken as the present $M$, and $M^{\prime}$ is replaced successively by $M_{1}, M_{2}, \cdots$, the integrands are bounded and converge to zero except possibly at a single point.

(ii) A fundamental sequence $\left\{M_{n}\right\}$ determining a point $M$ of $\Delta$ is $\rho$-convergent to $M$. This follows from the same argument as in (i) and the definition of a fundamental sequence.

(iii) $A$ sequence $\left\{M_{n}\right\}$ of points of $\Delta$ has a subsequence $\rho$-convergent to $a$ point $M$ of $\Delta$. Let $F_{1}, F_{2}, \cdots$ be an increasing sequence of bounded closed sets whose sum is $D$. Consider a fixed $M_{n}$. A fundamental sequence determining $M_{n}$ has at most a finite number of points in $F_{n}$. We can, by (ii), select from such a fundamental sequence a point which is not in $F_{n}$ and whose $\rho$-distance from $M_{n}$ does not exceed $1 / n$. Call this point $M_{n}^{\prime}$. We thus obtain a sequence $\left\{M_{n}^{\prime}\right\}$ of points of $D$ which, since $M_{n}^{\prime}$ is not in $F_{n}$, can have no accumulation point in $D$, and for which $\rho\left(M_{n}, M_{n}^{\prime}\right) \leqq 1 / n$. A subsequence of $\left\{M_{n}^{\prime}\right\}$ is fundamental, and determines an $M$ in $\Delta$. Application of the last inequality, the triangular inequality, and (ii) shows that the corresponding subsequence of $\left\{M_{n}\right\}$ is $\rho$-convergent to $M$.

(iv) Any sequence $\left\{M_{n}\right\}$ of points of $\mathcal{D}$ has a subsequence $\rho$-convergent to a point $M$ of $\mathcal{D}$. If an infinity of points of $\left\{M_{n}\right\}$ are in $D$, and these have an accumulation point $M$ there, a subsequence consists of points of $D$ and converges to $M$. We then apply (i). If an infinity of points of $\left\{M_{n}\right\}$ are in $D$ but there is no point of accumulation there, a subsequence is fundamental, and we apply (ii). This leaves only the possibility that an infinity of the $M_{n}$ are in $\Delta$; (iii) applies here.

(v) A sequence $\left\{M_{n}\right\}$ of points of $D$ which is $\rho$-convergent to a point $M$ of $D$ is convergent in the ordinary sense to $M$. For, if the sequence $\left\{M_{n}\right\} \operatorname{did}$ not converge to $M$, it would either have a subsequence convergent to an $M^{\prime} \neq M$ in $D$, or would have a fundamental subsequence. In the first case (i), and in the second (ii), would imply a contradiction.

The various statements of the theorem now follow at once. (iv), as the statement of self-compactness, implies completeness and compactness. That $\Delta$ is $\rho$-closed follows from (iii). $D$, as the complement of $\Delta$ in $\mathcal{D}$, is $\rho$-open. From (ii) and the fact that a fundamental sequence consists only of points of $D$, it follows that every $\rho$-neighborhood of a point of $\Delta$ contains points of $D$. The equivalence of the two topologies in $D$ is a consequence of (i) and (v).

Theorem II. The function $K(M, P)$, for fixed $P$, is $\rho$-continuous as a func- 
tion of $M$ in $\mathcal{D}$, except at $M=P$. More generally, if $F$ is a bounded closed subset of $D$ and if $G$ is a $\rho$-closed subset of $D-F$, then $K(M, P)$ is uniformly continuous in both arguments for $M$ in $G$ and $P$ in $F$.

The first statement, when $M$ is in $D$, is a consequence of the equivalence of continuity and $\rho$-continuity in $D$. Suppose that $M$ is in $\Delta$, and let $\left\{M_{n}\right\}$ be any sequence of points of $\mathcal{D} \rho$-convergent to $M$. For a subsequence $\left\{M_{n}^{\prime}\right\}$ of $\left\{M_{n}\right\}$, we have $K\left(M_{n}^{\prime}, P\right) \rightarrow v(P)$, where $v(P)$ is harmonic in $D$. If in (2.1) $M$ is taken as the present $M$ and $M^{\prime}$ is replaced successively by $M_{1}^{\prime}, M_{2}^{\prime}, \cdots$, then the condition $\rho\left(M, M_{n}^{\prime}\right) \rightarrow 0$ implies that the integral in (2.1) with $K\left(M^{\prime}, P\right)$ replaced by $v(P)$ has the value zero. Thus $v(P)=K(M, P)$ for all $P$ in $\Sigma$, and harmonic continuation extends this equality to all $P$ in $D$. In other words, $\left\{M_{n}\right\}$ has a subsequence $\left\{M_{n}^{\prime}\right\}$ such that $K\left(M_{n}^{\prime}, P\right) \rightarrow K(M, P)$. Since the same argument applies to any subsequence of $\left\{M_{n}\right\}$, it follows that $K\left(M_{n}, P\right) \rightarrow K(M, P)$.

For the second statement, consider the $K(M, P)$ with $M$ in $G$ as a family of harmonic functions of $P$ in $D-G$. They form a family uniformly bounded near any point of $D-G$; thus, since harmonic, they are equicontinuous at any such point, in particular at any point of $F$. Hence, for $M$ in $G$ and $P$ in $F$, $K(M, P)$ is continuous in $M$ and continuous in $P$ uniformly in $M$. Continuity in both arguments follows from this and the compactness of the ranges of $M$ and $P$.

The notions of $\rho$-closed and $\rho$-open sets arising from the metric $\rho$ in $\mathscr{D}$ extend in a familiar manner to that of a $\rho$-Borel set. A system of sets in a space is customarily called a Borel field if it contains the empty set, contains with each of its sets the complement, and contains with each sequence of its sets the $\operatorname{sum}\left({ }^{20}\right)$. The system of $\rho$-Borel sets of $\mathcal{D}$ is defined as the smallest Borel field consisting of sets in $\mathcal{D}$ which contains all $\rho$-open sets.

Theorem III. The $\rho$-Borel subsets of $D$ are identical with the subsets of $D$ measurable Borel in the ordinary sense.

Consider the system $\subseteq$ of sets of $\mathcal{D}$ whose intersection with $D$ is a Borel set. $\mathfrak{S}$ is clearly a Borel field. Since the intersection of a $\rho$-open set with $D$ is $\rho$-open and, hence, by Theorem $\mathrm{I}$, open, $\subseteq$ contains all $\rho$-open sets, and thus all $\rho$-Borel sets. It follows that every $\rho$-Borel subset of $D$ is Borel.

Conversely, a similar consideration of the system $\mathfrak{S}^{\prime}$ of all sets in space whose intersection with $D$ is $\rho$-Borel shows that $\boldsymbol{S}^{\prime}$ contains all Borel sets in space. In particular, every Borel subset of $D$ is $\rho$-Borel.

Relative to any Borel field there are definable the notions of a completely additive function of sets of the field, of measurability of a point function with respect to the field, and of an integral of a measurable point function with

(20) See, for example, S. Saks, Theory of the Integral, 2d revised edition, translated by L. C. Young, Warsaw, 1937, p. 7. Saks uses the term additive class. 
respect to a completely additive function of $\operatorname{sets}\left({ }^{21}\right)$. In particular, we shall make use of these notions relative to the system of $\rho$-Borel sets. Since $\mathcal{D}$ is $\rho$-compact, the theory of such an integral does not differ essentially from that a Radon-Stieltjes-Lebesgue integral over a bounded closed portion of Euclidean space.

We shall have occasion to use only those completely additive set functions which are finite and non-negative, and shall use the terms mass distribution or mass function only in reference to such. The notion of weak convergence of a sequence of mass distributions is of considerable importance, and may be recalled. If $F$ is a closed and compact set in a metric space, a distribution $\mu(e)$ over $F$ is called the weak limit of a sequence $\left\{\mu_{n}(e)\right\}$ of distributions over $F$, if the condition

$$
\lim _{n \rightarrow \infty} \int_{F} f(S) d \mu_{n}\left(e_{S}\right)=\int_{F} f(S) d \mu\left(e_{S}\right)
$$

holds for every function $f(S)$ which is continuous over $F$. The most important feature of weak convergence is the

Selection theOREm( $\left.{ }^{22}\right)$. A sequence of distributions over a closed compact set $F$ in which the total masses are uniformly bounded has a subsequence weakly convergent to a distribution over $F$.

3. The function $u_{A}(P)$ and the representation. Throughout this section and the next we shall employ the metric $\rho$ in $\mathcal{D}$. Thus, the terms, open, closed, distance, etc., will be understood, in the absence of specific mention to the contrary, in the sense of this metric. If $G$ is any set in $\mathcal{D}$, we shall denote by $[G]$ the intersection of $D$ with the $\rho$-closure of $G$. $[G]$ is a relatively closed subset of $D$ in the sense of the ordinary topology of $D$.

(21) The integral most convenient for our present purposes is one of Stieltjes type; that is, one whose dependence upon a mass distribution is explicit. The theory in the extended form needed here may be found in Saks, op. cit.

(22) The definition of weak convergence given above is copied after that of J. Radon, Theorie und Anwendungen der absolut additiven Mengenfunktionen, Sitzungsberichte der Akademie der Wissenschaften, Vienna, 1913, p. 1337. All that is really essential for the extension of the result is the observation that, since we are in a metric space and $F$ is compact, there is a countable neighborhood system covering $F$. It may be noted that the condition (2.2) is equivalent to three: (i) the $\mu_{n}(F)$ are bounded independently of $n$; (ii) $\lim \inf _{n \rightarrow \infty} \mu_{n}(e) \geqq \mu(e)$, for subsets $e$ open in $F$; (iii) $\lim \sup _{n \rightarrow \infty} \mu_{n}(e) \leqq \mu(e)$, for closed subsets $e$ of $F$. It is possible to imbed the countable neighborhood system of $F$ in a system of sets $\mathfrak{B}$, also countable, containing with each of its sets the complement in $F$, and with each pair of its sets the sum and intersection. By the "diagonal process" we can determine a subsequence of the mass distributions having a limit $\lambda(p)$ for each set $p$ of $\mathfrak{B} . \lambda(p)$ is additive in the restricted sense for sets in $\mathfrak{B}$, and can be used to generate a Carathéodory outer measure expressible in a familiar fashion in terms of coverings by sequences of sets from $\mathfrak{B}$. The measure function of this outer measure, when restricted to Borel sets, satisfies the requirements (ii), and (iii) for $\mu(e)$. 
Definition 1. Let $u(P)$ be a non-negative harmonic function in $D$, and let $A$ be a closed subset of $\Delta$. The function $u_{A}(P)$ is defined as the greatest lower bound of $u_{[G]}^{*}(P)$ as $G$ ranges over all open sets containing $A$.

Lemma 1. Let $u(P)$ and $A$ be as in the above definition. Let $G_{1}, G_{2}, \cdots$ be a descending sequence of open sets which contain $A$ and whose closures have $A$ as their intersection. Then $u_{\left[G_{n}\right]}^{*}(P) \downarrow u_{A}(P)$.

Obviously, the sets $\left[G_{n}\right]$ form a descending sequence having a void intersection. The functions $u_{\left[G_{n}\right]}^{*}(P)$ form a descending sequence and, from some $n$ on, are non-negative harmonic in any bounded closed subset of $D$. Thus, $u_{\left[{ }^{*} G_{n}\right]}(P) \downarrow v(P)$, where $v(P)$ is non-negative harmonic in $D$. From Definition 1 , we have $v(P) \geqq u_{A}(P)$. On the other hand, corresponding to any point $P$ and any positive $\epsilon$, there is an open set $G$ containing $A$ such that, for this particular $P$, we have $u_{\left[{ }^{G}\right]}^{*}(P) \leqq u_{A}(P)+\epsilon$. But, since $G$ will contain all but a finite number of the sets $G_{n}$, we have $u_{[G]}^{*}(P) \geqq v(P)$. Combining these inequalities and using the arbitariness of $\epsilon$, we obtain $v(P)=u_{A}(P)$.

We now derive a number of elementary properties of the function $u_{A}(P)$. In the statement of the following theorem, $u(P)$ and $v(P)$ will denote nonnegative harmonic functions in $D ; A, B$, etc. will be closed subsets of $\Delta$.

Theorem I. The function $u_{A}(P)$ is non-negative harmonic in $D$. It has the following properties $\left({ }^{23}\right)$ :

(a) $u(P) \geqq u_{A}(P)$ for all $P$ in $D$.

(b) If $u(P) \geqq v(\dot{P})$ for all $P$ in $D$, then $u_{A}(P) \geqq v_{A}(P)$.

(c) $(u+v)_{A}(P)=u_{A}(P)+v_{A}(P)$.

(d) $(c \cdot u)_{A}(P)=c \cdot u_{A}(P)$, where $c$ is a non-negative number.

(e) $u_{\Delta}(P)=u(P)$.

(f) If $A \supseteqq B$, then $\left(U_{B}\right)_{A}(P)=u_{B}(P)$.

(g) If $A \supseteqq B$, then $u_{A}(P) \geqq u_{B}(P)$. More generally, if $A_{n} \downarrow A$, then $u_{A_{n}}(P)$ $\downarrow u_{A}(P)$.

(h) $u_{(A+B)}(P) \leqq u_{A}(P)+u_{B}(P)$.

That $u_{A}(P)$ is harmonic was shown incidentally in the proof of Lemma 1 . Statements (a), (b), (c), and (d) are immediate consequences of Lemma 1 and the corresponding statements in Theorem II of $\$ 1$.

To prove (e), suppose that $G$ is an open set containing $\Delta . \mathcal{D}-G$ is a subset of $D$, is $\rho$-closed and, therefore, is closed in the ordinary sense. It is also bounded. If it were not, there could be selected from it a sequence of points having no point of accumulation in $D$; this sequence would contain a fundamental subsequence determining a point of $\Delta$. This is impossible. From this it follows that $D-[G]$ is a bounded open set completely interior to $D$. In

${ }^{(23)}$ Several of the statements here are shown by Theorem III, $\$ 4$, and its Corollary 3 to admit of considerable strengthening. 
every boundary point of $D-[G], u(P)$ is harmonic; thus it is determined in $D-[G]$ by its own boundary values. It follows that in $D-[G], u_{[G]}^{*}(P)=u(P)$. (e) now follows if we let $G$ close down upon $\Delta$ through a sequence of the type prescribed in the statement of Lemma 1.

To prove (f), let $G$ and $H$ be open sets containing $A$ and $B$ respectively, and assume that $G \supseteqq H$. From (f) of Theorem II, $\$ 1$, we have $\left(u_{[H]}^{*}\right)_{[G]}^{*}(P)=u_{[H]}^{*}(P)$. If we keep $G$ fixed and allow $H$ to run through a descending sequence of sets closing down upon $B$ as in Lemma 1 , we obtain, with the aid of (e) of Theorem II, $\S 1$ (using $u(P)$ for a majorant), $\left(u_{B}\right)_{[G]}^{*}(P)=u_{B}(P)$ for all $P$ in $D-[G]$. (f) now follows as the limiting form of this last relation when $G$ is allowed to run through a descending sequence of sets closing down upon $A$.

The first statement of $(\mathrm{g})$ follows from Definition 1 and the fact that any open set containing $A$ also contains $B$. For the more general statement, the functions $u_{A_{n}}(P)$ form a descending sequence having a harmonic limit $v(P)$, which clearly must dominate $u_{A}(P)$. On the other hand, since any open set $G$ containing $A$ contains all but a finite number of the $A_{n}$, we have, for such $G$, $u_{[G]}^{*}(P) \geqq v(P)$. It follows that $u_{A}(P) \geqq v(P)$, and therefore that $u_{A}(P)$ $=v(P)$.

To prove (h), let $G$ and $H$ be open sets containing $A$ and $B$ respectively. From (h) of Theorem II, $\$ 1$, we have, writing $K=G+H$ and observing that $[K]=[G]+[H], u_{[K]}^{*}(P) \leqq u_{[G]}^{*}(P)+u_{[H]}^{*}(P)$. If now $G$ and $H$ run simultaneously through descending sequences of sets closing down respectively upon $A$ and $B, K$ runs through a sequence closing down upon $A+B$, and (h) follows as the limiting form of the last inequality.

Theorem II. If $u(P)$ is non-negative harmonic in $D$, and $A$ is a closed subset of $\Delta$, then there exists a mass distribution $\mu_{A}(e)$ over $A$ such that

$$
u_{A}(P)=\int_{A} K(M, P) d \mu_{A}\left(e_{M}\right)
$$

for all $P$ in $D$. The total mass, $\mu_{A}(A)$, is equal to the value of the function $u_{A}(P)$ at the point $P_{0}$.

It should be remarked that the uniqueness of the distribution $\mu_{A}(e)$ is not asserted in this statement. As we shall see later, there may actually be more than one distribution satisfying the requirements of the theorem.

Let $G$ be an open set containing $A$ and having the point $P_{0}$ as an exterior point. Denote by $\bar{G}$ the $\rho$-closure of $G$. Let $\sigma$ be a closed subset of $[G] . \sigma$ is then closed and bounded (cf. the proof of part (e) of Theorem I of the present section) in the ordinary topology of $D$. If we now transform the integral of (1.2), and use the fact that $G\left(M, P_{0}\right)$ as a function of $M$ in $\sigma$ is continuous and positive, we obtain 


$$
\begin{aligned}
u_{\sigma}^{*}(P) & =\int_{\sigma} G(M, P) d \nu_{\sigma}\left(e_{M}\right) \\
& =\int_{\sigma} K(M, P) \cdot G\left(M, P_{0}\right) d \nu_{\sigma}\left(e_{M}\right)=\int_{\sigma} K(M, P) d \mu_{\sigma}\left(e_{M}\right),
\end{aligned}
$$

in which

$$
\mu_{\sigma}(e)=\int_{\theta} G\left(M, P_{0}\right) d \nu_{\sigma}\left(e_{M}\right)
$$

Since $\mu_{\sigma}(e)$ is a Borel mass distribution vanishing outside $\sigma$, it may, by the result of Theorem III of $\$ 2$, equally well be interpreted as a $\rho$-Borel mass distribution over $\sigma$. Since $\sigma \subset \bar{G}$, the result of (3.2) may be written

$$
u_{\sigma}^{*}(P)=\int_{\bar{G}} K(M, P) d \mu_{\sigma}\left(e_{M}\right)
$$

The total mass of $\mu_{\sigma}(e)$, calculated by writing $P=P_{0}$ in (3.3) and recalling that $K\left(M, P_{0}\right) \equiv 1$, is $u_{\sigma}^{*}\left(P_{0}\right)$, and thus does not exceed $u\left(P_{0}\right)$.

If now $\sigma$ is allowed to run through an ascending sequence of sets whose sum is $[G]$, then, since the $\mu_{\sigma}(e)$ have total masses not exceeding $u\left(P_{0}\right)$ and lying in the closed compact set $\bar{G}$, we have for some subsequence of $\left\{\mu_{\sigma}(e)\right\}-$ and we may assume it already extracted-a weak limit distribution $\mu_{\tilde{\sigma}}(e)$ having its total mass (also not greater than $u\left(P_{0}\right)$ ) in $\bar{G}$. From Theorem II, (g), $\$ 1$, we have $u_{\sigma}^{*}(P) \uparrow u_{[G]}^{*}(P)$. Using the weak convergence of the distributions and the continuity of $K(M, P)$ as a function of $M$ in $\bar{G}$ when $P$ is in $D-[G]$, we obtain as the limiting form of the equation (3.3)

$$
u_{[G]}^{*}(P)=\int_{\bar{G}} K(M, P) d \mu_{\bar{G}}\left(e_{M}\right) \quad(P \text { in } D-[G]) .
$$

Now allow $G$ to run through a sequence closing down upon $A$ in the manner prescribed in the statement of Lemma 1. From the result of Lemma 1, by an argument similar to that of the preceding paragraph coupled with the observation that a weak limiting distribution, $\mu_{A}(e)$, of the $\mu_{G}(e)$ must have its total mass in every $\bar{G}$ and thus in $A$, we obtain (3.1) as the limiting form of (3.4). The last statement of the theorem is immediate if we put $P=P_{0}$ in (3.1).

We now have a representation theorem:

Theorem III. If $u(P)$ is non-negative harmonic in $D$, then there exists a distribution $\mu(e)$ over $\Delta$ such that

$$
u(P)=\int_{\Delta} K(M, P) d \mu\left(e_{M}\right),
$$

for all $P$ in $D$. Conversely, for any distribution $\mu(e)$ over $\Delta$, the integral in (3.5) 
represents a non-negative harmonic function $u(P)$. The total mass, $\mu(\Delta)$, is equal to the value of $u(P)$ at the point $P_{0}$.

The first and last statements follow as corollaries of Theorem I, (e), and Theorem II of the present section if $A$ is taken as $\Delta$ in the latter theorem.

For the converse statement, since $K(M, P)$ is continuous as a function of $M$ in $\Delta$, the integral in (3.5) can be approximated by means of Riemann sums. Indeed, in view of Theorem II, $\$ 2$, the approximation is uniform for $P$ in any bounded closed subset of $D$. The approximating sums are finite positive linear combinations of $K(M, P)$ 's with $M$ in $\Delta$, and, as such, are non-negative harmonic. The result is now immediate.

4. The minimal functions and the uniqueness problem. As has been indicated before, the representation obtained in the preceding section fails to give a complete determination of the distribution in terms of which a specified harmonic function is represented. In this section we shall obtain a characterization of the minimal harmonic functions and establish the existence of a unique canonical representation in terms of these functions.

Lemma 1. Suppose that $u(P)$ is positive harmonic and minimal. Let $A$ be any $\rho$-Borel subset of $\Delta$. If now a relation of the form

$$
u(P) \geqq \int_{A} K(M, P) d \mu\left(e_{M}\right)>0
$$

obtains for all $P$ in $D$, then $u(P)=u\left(P_{0}\right) \cdot K(S, P)$, where $S$ is some point in $A$.

$\mu(A)$ is positive, as is easily seen by setting $P=P_{0}$ in (4.1). $A$, therefore, has a closed subset $A_{1}$ for which $\mu\left(A_{1}\right)$ is positive. $A_{1}$, being compact, can be covered by a finite number of its closed subsets, all of them having diameter less than some selected positive number. At least one such subset has a positive $\mu$ mass. We select a particular such and call it $A_{2}$. By proceeding in this way inductively, it is possible to construct a descending sequence $A_{1}, A_{2}$, . . of closed subsets of $A$ whose diameters approach zero and each of which has a positive $\mu$ mass. Let $S$ be the (unique) point common to all the $A_{n}$.

Now since $\mu\left(A_{n}\right)$ is positive, the integral in (4.1) extended over $A_{n}$ instead of $A$ represents a positive harmonic function dominated by the minimal function $u(P)$, and is thus equal to $c_{n} \cdot u(P)$, where $c_{n}$ is positive. If we write $\mu_{n}(e) \equiv c_{n}^{-1} \cdot \mu\left(A_{n} \cdot e\right)$, there follows

$$
u(P)=\int_{A_{n}} K(M, P) d \mu_{n}\left(e_{M}\right) .
$$

The total mass of the distribution $\mu_{n}(e)$ is $u\left(P_{0}\right)$ and is located upon $A_{n}$. Thus the $\mu_{n}(e)$ have as weak limit a point mass of amount $u\left(P_{0}\right)$ located at $S$. The relation $u(P)=u\left(P_{0}\right) \cdot K(S, P)$ now follows as the limiting form of (4.2). 
CoRollary 1. Every minimal positive harmonic function in $D$ is a positive multiple of some $K(S, P)$, where $S$ is in $\Delta$.

This follows on taking $A=\Delta$ in the lemma and recalling the result of Theorem III of the preceding section.

Corollary 2. If $K(S, P)$ is minimal and $A$ is a closed subset of $\Delta$ such that $K_{A}(S, P)$ is positive, then $S$ is in $A$.

Since $K(S, P) \geqq K_{A}(S, P)$, Theorem II of the preceding section with $A$ taken as the present $A$ implies a relation of the form (4.1).

Definition 1. We define the function $\psi(S)$ for $S$ in $\Delta$ as $K_{\{S\}}\left(S, P_{0}\right)$; that is, as the value at $P_{0}$ of $u_{A}(P)$, where $u(P)$ is $K(S, P)$ and $A$ is the set consisting of the single point $S$.

TheOREM I. The function $\psi(S)$ has only the two possible values 1 and 0 . The function $K(S, P)$ is minimal or not according as $\psi(S)$ is equal to 1 or 0 .

Theorem II of the preceding section with $A=\{S\}$ has the following consequence:

$$
u_{\{S\}}(P)=\int_{\{S\}} K(M, P) d \mu\{S\}\left(e_{M}\right)=u_{\{S\}}\left(P_{0}\right) \cdot K(S, P) .
$$

In particular, putting $u(P)=K(S, P)$, we get

$$
K_{\{S\}}(S, P)=\psi(S) \cdot K(S, P) .
$$

Application to this of (f) and (d) Theorem I, §3, yields

$$
K_{\{S\}}(S, P)=\left(K_{\{S\}}\right)_{\{S\}}(S, P)=\psi(S) \cdot K_{\{S\}}(S, P) .
$$

If we put $P=P_{0}$, this yields $\psi(S)=[\psi(S)]^{2}$, which proves that $\psi(S)=1$ or 0 .

Assume now that $\psi(S)=1$. We show that this implies that $K(S, P)$ is minimal. Let $u(P)$ be any positive harmonic function dominated by $K(S, P)$. Write $v(P)=K(S, P)-u(P)$. Then $v(P)$ is non-negative harmonic. Now

$$
u(P) \geqq u_{\{s\}}(P), \quad v(P) \geqq v\{s\}(P),
$$

and

$$
\begin{aligned}
K(S, P) & =u(P)+v(P) \geqq u_{\{S\}}(P)+v_{\{S\}}(P) \\
& =K\{S\} \\
& (S, P)=\psi(S) \cdot K(S, P)=K(S, P) .
\end{aligned}
$$

But clearly (4.5) can hold only if equality obtains in both of the relations (4.4). In particular, $u(P)=u_{\{S\}}(P)$; from (4.3) the latter function is a multiple of $K(S, P)$. Thus $K(S, P)$ is minimal.

Assume, conversely, that $K(S, P)$ is minimal. Suppose that $A$ is a closed subset of $\Delta$ having $S$ as an interior point relative to $\Delta$; let $B$ be the closure 
of the complement of $A$ in $\Delta$. We now have $K_{B}(S, P)=0$; for, if not, Corollary 2 above would imply that $S$ is in $B$. Thus,

$$
\begin{aligned}
K(S, P) & =K_{\Delta}(S, P)=K_{(A+B)}(S, P) \\
& \leqq K_{A}(S, P)+K_{B}(S, P)=K_{A}(S, P) \leqq K(S, P) ;
\end{aligned}
$$

that is, $K_{A}(S, P)=K(S, P)$. If now $A$ is allowed to run, for example, through the sequence $\left\{A_{n}\right\}$, where $A_{n}$ is the set of points of $\Delta$ whose $\rho$-distance from $S$ does not exceed $1 / n$, then $A_{n} \downarrow\{S\}$, and we have, as the limiting form of the result just obtained, $K_{\{S\}}(S, P)=K(S, P)$. On writing $P=P_{0}$ in this, there follows $\psi(S)=1$.

Definition 2. We shall denote by $\Delta_{0}$ and $\Delta_{1}$ respectively the sets of points of $\Delta$ for which $\psi(S)$ has the value 0 and the value 1 .

ThEOREM II. The set $\Delta_{0}$ is either void, or closed, or an $F_{\sigma}$.

We introduce an auxiliary sequence of sets $\Gamma_{n}$, where $n=1,2, \cdots$. The set $\Gamma_{n}$ is defined as the set (possibly void) of all points $S$ of $\Delta$ having the following property: If $G$ is any open set in $\mathcal{D}$ containing $S$ and having a $\rho$-diameter less than $1 / n$, then $K_{[G]}^{*}\left(S, P_{0}\right) \leqq \frac{1}{2}$. It is clear, incidentally, from this definition that the $\Gamma_{n}$ form an ascending sequence.

Since $K(S, P)$ is continuous as a function of $S$ in $\Delta$, it follows from the second statement in (e) of Theorem II, $\$ 1$ that for any open set $G$ the function $K_{[G]}^{*}\left(S, P_{0}\right)$ is lower semicontinuous as a function of $S$. In particular, if $S_{0}$ is a limit point of $\Gamma_{n}$ (assumed non-void) and $G$ is an open set of diameter less than $1 / n$ containing $S_{0}$, then the function $K_{[G]}^{*}\left(S, P_{0}\right)$ has a value not exceeding $\frac{1}{2}$ at points $S$ of a sequence approaching $S_{0}$. It follows that $S_{0}$ is in $\Gamma_{n}$; that is, $\Gamma_{n}$ is closed.

Now let $S$ be a point of $\Gamma_{n}$. Select an open set $G$ (e.g., an open $\rho$-sphere) containing $S$ and having a diameter less than $1 / n$. There follows $\psi(S)$ $=K_{\{S\}}\left(S, P_{0}\right) \leqq K_{[G]}^{*}\left(S, P_{0}\right) \leqq \frac{1}{2}<1$; that is, $\psi(S)=0$. This proves that $\Gamma_{n} \subseteq \Delta_{0}(n=1,2, \cdots)$.

Assume, conversely, that $S$ is in $\Delta_{0}$. Denote by $G_{n}$ the set of points of $\mathscr{D}$ whose distance from $S$ is less than $1 / n$. Then, since $\bar{G}_{n} \downarrow\{S\}$, we have $K_{\left[G_{n}\right]}^{*}\left(S, P_{0}\right) \downarrow K_{\{S\}}\left(S, P_{0}\right)=\psi(S)=0$. Hence, we may choose $n=m$ so great that $K_{\left[G_{m}\right]}^{*}\left(S, P_{0}\right) \leqq \frac{1}{2}$. Any open set $G$ which contains $S$ and has a diameter less than $1 / m$ is contained in $G_{m}$. Since for any such $G, K_{[G]}^{*}\left(S, P_{0}\right)$ $\leqq K_{\left[G_{m}\right]}^{*}\left(S, P_{0}\right)$, it follows that $S$ is in $\Gamma_{m}$.

The result of the last two paragraphs is to the effect that $\Delta_{0}$ is identical with the sum of the $\Gamma_{n}$, which were proved above to be closed or void( $\left.{ }^{24}\right)$.

(24) It seems a reasonable conjecture that the sets $\Gamma_{n}$ are nowhere dense in $\Delta$; thus that $\Delta_{0}$, actually, is of the first category in $\Delta$. An answer to this question would have interesting consequences. 
The particular consequence of the theorem just proved, that $\Delta_{0}$ is a $\rho$-Borel set, is needed in

Definition 3. A distribution $\mu(e)$ over $\Delta$ will be called canonical if $\mu\left(\Delta_{0}\right)=0$. $A$ representation of the form given by Theorem III, §3, is a canonical representation if the distribution occurring in it is canonical.

In a canonical distribution the total mass is carried on the set $\Delta_{1}$. Thus a canonical representation is one which involves, in a sense, only minimal $K(S, P)$ 's. It is our purpose to show that every non-negative harmonic function in $D$ has exactly one canonical representation. Before proceeding to this result it is convenient to prove a number of lemmas.

LEMma 2. If $\Gamma_{n}$ is one of the auxiliary sets introduced in the proof of Theorem II, then $u_{\Gamma_{n}}(P)=0$, for any function $u(P)$ positive harmonic in $D$.

The set $\Gamma_{n}$, being closed and compact, may be covered by a finite number of its closed subsets each of diameter less than $1 / n$. It is sufficient (Theorem I, (h), §3) to prove that $u_{A}(P)=0$ whenever $A$ is such a subset of $\Gamma_{n}$. $A$ being such a set, let $G$ be an open set also of diameter less than $1 / n$ containing $A$. From the defining property of $\Gamma_{n}$, we have $K_{[G]}^{*}\left(S, P_{0}\right) \leqq \frac{1}{2}$ for every $S$ in the set $A$.

Let $v(P)$ be a finite linear combination, with positive coefficients, of $K(S, P)$ 's with $S$ in $A$ :

$$
v(P)=\sum_{1}^{m} c_{\nu} \cdot K\left(S_{\nu}, P\right) \quad\left(c_{\nu}>0 ; S_{\nu} \text { in } A\right) .
$$

We then have

$$
\begin{aligned}
v_{[G]}^{*}\left(P_{0}\right) & =\sum_{1}^{m} c_{\nu} \cdot K_{[G]}^{*}\left(S_{\nu}, P_{0}\right) \leqq \frac{1}{2} \sum_{1}^{m} c_{\nu} \\
& =\frac{1}{2} \sum_{1}^{m} c_{\nu} \cdot K\left(S_{\nu}, P_{0}\right)=\frac{1}{2} v\left(P_{0}\right),
\end{aligned}
$$

for any $v(P)$ of the form (4.6).

More generally, suppose that the function $v(P)$ is expressible in the form of an integral:

$$
v(P)=\int_{A} K(M, P) d \mu\left(e_{M}\right)
$$

Approximation to this integral by means of Riemann sums yields an approximation to the $v(P)$ in (4.8) by a sequence of functions $v_{n}(P)$ of the form (4.6). Using the last statement in part (e) of Theorem II, $\$ 1$, and the result of (4.7) for the functions $v_{n}(P)$, we obtain 


$$
\begin{aligned}
v_{A}\left(P_{0}\right) & \leqq v_{[G]}^{*}\left(P_{0}\right) \leqq \liminf _{n \rightarrow \infty}\left(v_{n}\right)_{[G]}^{*}\left(P_{0}\right) \\
& \leqq \frac{1}{2} \lim _{n \rightarrow \infty} v_{n}\left(P_{0}\right)=\frac{1}{2} v\left(P_{0}\right)
\end{aligned}
$$

for any $v(P)$ of the form (4.8).

In particular, by Theorem II, $\S 3, u_{A}(P)$ is of the form (4.8). Hence, we have

$$
u_{A}\left(P_{0}\right)=\left(u_{A}\right)_{A}\left(P_{0}\right) \leqq \frac{1}{2} u_{A}\left(P_{0}\right) ;
$$

from which it follows that $u_{A}\left(P_{0}\right)=0$, thus that $u_{A}(P) \equiv 0$.

Lemma 3. Let $u(P)$ be positive harmonic in $D$, and let $\epsilon$ be an arbitrary positive number. Then there exists a closed subset $A$ of $\Delta_{1}$ such that $u\left(P_{0}\right) \leqq u_{A}\left(P_{0}\right)+\epsilon$. The set $A$ depends, of course, upon $u(P)$ and $\epsilon$.

Denote by $\Gamma_{m, n}(m, n=1,2, \cdots)$ the set of points of $\Delta$ whose distance from $\Gamma_{n}$ does not exceed $1 / m$. The sets $\Gamma_{m, n}$ are closed, and, for $n$ fixed, $m \rightarrow \infty$, we have $\Gamma_{m, n} \downarrow \Gamma_{n}$. As a consequence of Theorem I, $(\mathrm{g}), \S 3$, and the result of the preceding lemma, we may, for each $n$, choose $m=m(n)$ so great that if $B_{n}=\Gamma_{m(n), n}$, then $u_{B_{n}}\left(P_{0}\right)<2^{-n} \cdot \epsilon$. Having for each $n$ selected $B_{n}$ in this fashion, define $C_{n}$ as $B_{1}+B_{2}+\cdots+B_{n}$. The sets $C_{n}$ are closed and form with increasing $n$ an increasing sequence. Denote by $A_{n}$ the closure of the complement on $C_{n}$ in $\Delta$. The distance of the sets $A_{n}$ and $\Gamma_{n}$ is at least $1 / m(n)$; thus the $A_{n}$, which form a descending sequence, have an intersection $A$ which is closed and, having no point in common with any $\Gamma_{n}$, is a subset of $\Delta_{1}$.

We show that this $A$ satisfies the requirements of the lemma. By the con. struction above,

$$
u_{C_{n}}\left(P_{0}\right) \leqq \sum_{1}^{n} u_{B_{\nu}}\left(P_{0}\right)<\sum_{1}^{n} 2^{-\nu} \cdot \epsilon<\epsilon
$$

Observing that $A_{n}+C_{n}=\Delta$ and using (e) and (h) from Theorem I, $\S 3$, we obtain from this

$$
u\left(P_{0}\right)=u_{\Delta}\left(P_{0}\right)=u\left(A_{n}+C_{n}\right)\left(P_{0}\right) \leqq u_{A_{n}}\left(P_{0}\right)+u_{C_{n}}\left(P_{0}\right) \leqq u_{A_{n}}\left(P_{0}\right)+\epsilon .
$$

The limiting form of this inequality as $n$ becomes infinite, calculated with the aid of $(\mathrm{g})$ from the theorem just cited, is the inequality of the lemma.

Lemma 4. Let $A$ and $B$ be closed subsets of $\Delta$ having no common point. Assume that $B$ is a subset of $\Delta_{1}$ and that $\epsilon$ is an arbitrary positive number. Then there exists an open set $G$ containing $A$ such that for every $S$ of $B, K_{[G]}^{*}\left(S, P_{0}\right)<\epsilon$.

Let $G_{1}, G_{2}, \ldots$ be a descending sequence of open sets which have $P_{0}$ as an exterior point, which contain $A$, and whose closures have $A$ as their intersection. If the present lemma were false, we could find for each $n$ a point $S_{n}$ 
of $B$ and a number $\delta$, positive and independent of $n$, such that $K_{\left[{ }^{*} G_{n}\right]}\left(S_{n}, P_{0}\right)$ $\geqq \delta$. We show that this leads to a contradiction.

Using equation (3.4) from the proof of Theorem II, $\$ 3$, with $u(P)$ and $G$ there as the present $K\left(S_{n}, P\right)$ and $G_{n}$, we have

$$
K_{\left[G_{n}\right]}^{*}\left(S_{n}, P\right)=\int_{\vec{G}_{n}} K(M, P) d \mu_{n}\left(e_{M}\right),
$$

where $\mu_{n}(e)$ is a distribution over $\bar{G}_{n}$ whose total mass, calculated by writing $P=P_{0}$ in the equation, must be between the numbers $\delta$ and 1 inclusive. It is now possible to extract a subsequence of the natural numbers such that the corresponding subsequence of these distributions converges to a weak limiting distribution $\mu_{0}(e)$ over $A$ having a total mass of at least $\delta$. Since $B$ is closed and compact, it is then possible to extract from this subsequence a second, such that the corresponding subsequence of $\left\{S_{n}\right\}$ converges to a point $S_{0}$ in $B$. We may assume that both these extractions have already been performed, so that

$$
\begin{aligned}
K\left(S_{0}, P\right) & =\lim _{n \rightarrow \infty} K\left(S_{n}, P\right) \leqq \limsup _{n \rightarrow \infty} K_{\left[G_{n}\right]}^{*}\left(S_{n}, P\right) \\
& =\lim _{n \rightarrow \infty} \int_{\bar{G}_{n}} K(M, P) d \mu_{n}\left(e_{M}\right)=\int_{A} K(M, P) d \mu_{0}\left(e_{M}\right) .
\end{aligned}
$$

Since the last integral is positive and since $K\left(S_{0}, P\right)$ is minimal, it follows from Corollary 2 that $S_{0}$ is in $A$. This is the desired contradiction.

Lemma 5. Let $A$ be a closed subset of $\Delta$, and E a Borel subset of $\Delta_{1}$ having no point in common with $A$. Let $u(P)$ be a harmonic function of the form

$$
u(P)=\int_{E} K(M, P) d \mu\left(e_{M}\right)
$$

We then have $u_{A}(P)=0$.

Assume first that $E=B$, where $B$ is as in the statement of Lemma 4. Let $v(P)$ be a finite linear combination with positive coefficients of $K(S, P)$ 's with $S$ in $B$ :

$$
v(P)=\sum_{1}^{m} c_{\nu} \cdot K\left(S_{\nu}, P\right) \quad\left(c_{\nu}>0 ; S_{\nu} \text { in } B\right) .
$$

Now, $\epsilon$ being an arbitrary positive number, let $G$ be the $G$ of Lemma 4 corresponding to it for the present $A$ and $B$. We then have (cf. (4.7) in the proof of Lemma 2)

$$
v_{[G]}^{*}\left(P_{0}\right)=\sum_{1}^{m} c_{\nu} \cdot K_{[G]}^{*}\left(S_{\nu}, P_{0}\right) \leqq \epsilon \cdot \sum_{1}^{m} c_{\nu}=\epsilon \cdot \sum_{1}^{m} c_{\nu} \cdot K\left(S_{\nu}, P_{0}\right)=\epsilon \cdot v\left(P_{0}\right)
$$


By the use of Riemann sums, any $u(P)$ of the form (4.10) (with $E=B$ ) can be approximated by a sequence of functions $v_{n}(P)$ of the form (4.11). There follows (cf. (4.9) above)

$$
u_{A}\left(P_{0}\right) \leqq u_{[G]}^{*}\left(P_{0}\right) \leqq \liminf _{n \rightarrow \infty}\left(v_{n}\right)_{[G]}^{*}\left(P_{0}\right) \leqq \lim _{n \rightarrow \infty} \epsilon \cdot v_{n}\left(P_{0}\right)=\epsilon \cdot u\left(P_{0}\right)
$$

Since $\epsilon$ is arbitrary, $u_{A}\left(P_{0}\right)=0$; hence, $u_{A}(P) \equiv 0$.

When $E$ is of a more general form, we may write $E=B+C$, where $B$ and $C$ are without common points, $B$ is as above, and $C$ has a $\mu$-mass smaller than a preassigned $\epsilon$. If now $u(P)$ is decomposed into two parts represented by the integral of (4.10) extended over the sets $B$ and $C$ respectively, then, by what has just been proved, the first of these contributes nothing to the value of $u_{A}\left(P_{0}\right)$, while the second contributes an amount less than $\epsilon$. It then follows as above that $u_{A}(P)=0$.

THEOREM III. Every non-negative harmonic function $u(P)$ in $D$ admits of exactly one canonical representation. The canonical distribution $\mu(e)$ representing $u(P)$ is characterized by the relation

$$
u_{A}(P)=\int_{A} K(M, P) d \mu\left(e_{M}\right)
$$

which holds for every closed subset $A$ of $\Delta$.

We prove first the existence of a canonical representation. Let $u(P)$ be non-negative harmonic, $\epsilon$ positive, and $A$ the $A$ of Lemma 3 for this $u(P)$ and this $\epsilon$. Consider the decomposition

$$
u(P)=u_{A}(P)+\left[u(P)-u_{A}(P)\right] .
$$

By the result of Theorem II, $\S 3$, the function $u_{A}(P)$ admits a representation in terms of a distribution whose total mass is in $A$, thus a canonical representation. The function $u(P)-u_{A}(P)$ is non-negative harmonic, and its value at $P_{0}$, by the inequality of Lemma 3, cannot exceed $\epsilon$. This means: Any nonnegative harmonic function in $D$ can be expressed as the sum of two, one of which admits a canonical representation, and the other of which has a value at $P_{0}$ smaller than a preassigned positive number.

Let $\epsilon_{1}, \epsilon_{2}, \ldots$ be a decreasing sequence of positive numbers having zero as limit. Starting with the given $u(P)$ effect the decomposition $u(P)=u_{1}(P)+u_{1}^{\prime}(P)$, where $u_{1}(P)$ admits a canonical representation, and where $u_{1}^{\prime}\left(P_{0}\right)<\epsilon_{1}$. Repeat the process for $u_{1}^{\prime}(P)$, writing $u_{1}^{\prime}(P)=u_{2}(P)+u_{2}^{\prime}(P)$, where $u_{2}(P)$ has a canonical representation, and where $u_{2}^{\prime}\left(P_{0}\right)<\epsilon_{2}$. Proceeding in this way we obtain recursively a sequence of decompositions:

$$
u(P)=u_{1}(P)+u_{1}^{\prime}(P), \quad u_{n-1}^{\prime}(P)=u_{n}(P)+u_{n}^{\prime}(P) \quad(n=2,3, \cdots),
$$


where $u_{n}(P)$ has a canonical representation, and where $u_{n}^{\prime}\left(P_{0}\right)<\epsilon_{n}$. Combining the first $m$ of these relations, we get

$$
u(P)=\sum_{1}^{m} u_{n}(P)+u_{m}^{\prime}(P) .
$$

Since the $u_{m}^{\prime}(P)$ form a decreasing sequence of non-negative harmonic functions vanishing in their limit at $P_{0}$, the limit vanishes identically and we have

$$
u(P)=\sum_{1}^{\infty} u_{n}(P) .
$$
Write

Now, for each $n$, let $\mu_{n}(e)$ be a canonical distribution representing $u_{n}(P)$.

$$
\mu(e)=\sum_{1}^{\infty} \mu_{n}(e) .
$$

Any distribution represented by a partial sum of this series, since it represents a harmonic function which does not exceed $u(P)$, has a total mass not exceeding $u\left(P_{0}\right)$. Hence, the series defines a finite distribution $\mu(e)$, which is obviously canonical. We now have

$$
\begin{aligned}
u(P) & =\sum_{1}^{\infty} u_{n}(P)=\sum_{1}^{\infty} \int_{\Delta} K(M, P) d \mu_{n}\left(e_{M}\right) \\
& =\int_{\Delta} K(M, P) d \mu\left(e_{M}\right),
\end{aligned}
$$

which completes the existence proof.

We prove the uniqueness by showing that the relation (4.12) holds for any canonical distribution $\mu(e)$ representing $u(P)$. This is sufficient, since (4.12), with $P=P_{0}$, yields $u_{A}\left(P_{0}\right)=\mu(A)$, and thus shows that $\mu(e)$ is determined for all closed, hence for all Borel, sets in $\Delta$.

Assume thus that $\mu(e)$ is a canonical distribution representing $u(P)$. For brevity we shall write

$$
u(E ; P)=\int_{E} K(M, P) d \mu\left(e_{M}\right),
$$

when $E$ is any Borel subset of $\Delta$. Since $\Delta$ and $\Delta_{1}$ differ by the $\mu$-null set $\Delta_{0}$, we have for such $E$

$$
u(E ; P)=u\left(\Delta_{1} E ; P\right) .
$$

Now let $A$ be a closed subset of $\Delta$. From the additivity of the integral as a function of sets there follows

$$
u(P)=u(\Delta ; P)=u\left(\Delta_{1} ; P\right)=u\left(\Delta_{1} A ; P\right)+u\left(\Delta_{1}-A ; P\right) .
$$


Applying to this result that of Lemma 5 with $E$ there taken as $\Delta_{1}-A$ and $A$ taken as the present $A$, we obtain

$$
u_{A}(P)=u_{A}\left(\Delta_{1} A ; P\right)+u_{A}\left(\Delta_{1}-A ; P\right)=u_{A}\left(\Delta_{1} A ; P\right) .
$$

Denote by $A_{n}(n=1,2, \cdots)$ the set of points of $\Delta$ whose distance from $A$ does not exceed $1 / n$, and by $B_{n}$ the closure of the complement of $A_{n}$ in $\Delta$. $A_{n}$ and $B_{n}$ are closed, $A_{n}+B_{n}=\Delta$, and $B_{n}$ has no point in common with $A$. With the aid of Lemma 5 with $E$ taken as $\Delta_{1} A$ and $A$ there taken as the present $B_{n}$, we have

$$
\begin{aligned}
u\left(\Delta_{1} A ; P\right) & =u_{\Delta}\left(\Delta_{1} A ; P\right)=u_{\left(A_{n}+B_{n}\right)}\left(\Delta_{1} A ; P\right) \\
& =u_{A_{n}}\left(\Delta_{1} A ; P\right)+u_{B_{n}}\left(\Delta_{1} A ; P\right)=u_{A_{n}}\left(\Delta_{1} A ; P\right) \leqq u\left(\Delta_{1} A ; P\right) .
\end{aligned}
$$

Since $A_{n} \downarrow A$, the limiting form of this is

$$
u_{A}\left(\Delta_{1} A ; P\right)=u\left(\Delta_{1} A ; P\right) \text {. }
$$

From (4.14), (4.15), and (4.13) follows

$$
u_{A}(P)=u_{A}\left(\Delta_{1} A ; P\right)=u\left(\Delta_{1} A ; P\right)=u(A ; P),
$$

which completes the proof.

CoRollary 3. The function $u_{A}(P)$, defined originally for closed subsets of $\Delta$, admits of extension to a completely additive function of Borel sets in $\Delta$.

COROLlary 4. The condition that $\Delta_{0}$ be void is both necessary and sufficient for the uniqueness in general $\left({ }^{25}\right)$ of the representation of Theorem III of the preceding section.

If $\Delta_{0}$ is void, all representations are canonical, and thus unique. On the other hand, if $S$ is a point of $\Delta_{0}, K(S, P)$ has at least two representations, viz., its canonical representation and its representation by a unit point-mass at $S$.

5. Examples and applications. We shall first clear up by an example the question of the existence of a domain for which the set $\Delta_{0}$ is non-void. Since a point of divergence of the representation in $\$ 3$ from the Poisson-Stieltjes integral formula lies in the (presumable) failure of that representation to be unique, and since much of the complication of $\$ 4$ occurs on this account, it is desirable to have this example to show that the difficulty is genuine, and not simply the result of an ineffectiveness of the particular analytical devices employed. To show further that the presence of the set $\Delta_{0}$ is not connected with any necessary complication in the topological structure of the domain, this

(25) It is a question of interest whether bounded positive harmonic functions can have any but canonical representations. This reduces to an investigation of the representations of $l(P) \equiv 1$. It may be remarked that the function $l_{A}(P)$ plays a role analogous to that of the sweptout mass function $m(e, P)$, and serves as a natural starting point for an investigation of the "Dirichlet problem" associated with the present notion of ideal boundary. 
example has been chosen so that the domain together with its (ordinary) boundary is the topological image of a closed sphere.

We require certain properties of the swept-out mass for a domain limited by a simple closed surface of bounded curvature $\left({ }^{26}\right)$. Such a domain may for our present purposes be characterized by the existence of a positive number $r$ (called here an admissible radius for the domain) such that each boundary point of the domain lies at the point of tangency of two spheres of radius $r$ having their interiors respectively interior and exterior to the domain. The boundary surface of such a domain necessarily has in each point a well defined normal varying continuously in direction from point to point, and has an area given by the elementary formula. The distribution $m(e, P)$ is absolutely continuous with respect to area, and its superficial density $\Omega(S, P)$ at a boundary point $S$ is positive, varies continuously with $S$, and is given by the Poisson formula

$$
\Omega(S, P)=\frac{1}{4 \pi} \cdot \frac{\partial}{\partial n_{S}} G(S, P),
$$

in which $n_{S}$ denotes the inward directed normal at the point $S$, and $G(M, P)$ is the Green's function. $\Omega(S, P)$ is positive harmonic in $P$, approaches zero as $P$ approaches a boundary point distinct from $S$, and admits the estimate

$$
\Omega(S, P) \leqq \frac{1}{2 \pi} \frac{\cos \phi}{\overline{S P^{2}}}+\frac{1}{4 \pi r \cdot \overline{S P}},
$$

where $\phi$ is the angle between the inward normal at $S$ and the directed segment $S P$, and where $r$ is an admissible radius for the domain.

Lemma 1. Let $D$ be a (bounded) domain whose boundary $d$ is of bounded curvature, and let $\delta$ be a positive number. There exist positive constants $k_{1}$ and $k_{2}$ such that

$$
k_{1} \cdot \operatorname{dist}(P, d) \leqq \Omega(S, P) \leqq k_{2} \cdot \operatorname{dist}(P, d),
$$

whenever $\overline{S P} \geqq \delta . k_{1}$ depends only upon the domain; $k_{2}$ depends only upon the domain and $\delta$.

Let $r$ be an admissible radius for $D$. Let $D_{1}$ be the set of points of $D$ whose distance from $d$ is at least $r . \Omega(S, P)$ has a positive lower bound $m$ taken over all $P$ in $D_{1}, S$ in $d$. Obviously, if $P$ is in $D_{1}, \Omega(S, P) \geqq(m / \operatorname{diam}(D)) \cdot \operatorname{dist}(P, d)$. Suppose that $P=P_{1}$ is a point of $D$ not in $D_{1}$. Let $S_{1}$ be the closest point of $d$ to $P_{1}$. Let $\Sigma_{1}$ be the sphere of radius $r$ internally tangent to $d$ at $S_{1}$, and let $Q_{1}$

${ }^{(26)}$ See de la Vallee Poussin, loc. cit. $\left.{ }^{1}\right)$. The definition used here is equivalent to that of the cited reference for domains with simple boundaries. It may be remarked, however, that the largest admissible radius in the sense used here is not necessarily identical with the minimum radius of curvature of the boundary surface. 
be its center. $Q_{1}$ is in $D_{1}$, and $Q_{1}, P_{1}, S_{1}$ lie in that order along the inward normal at $S_{1}$. Using the inequality $\Omega\left(S, Q_{1}\right) \geqq m$, and the Harnack inequality for the sphere $\Sigma_{1}$, we obtain $\Omega\left(S, P_{1}\right) \geqq(m / 4 r) \cdot \overline{P_{1} S_{1}}=(m / 4 r) \cdot \operatorname{dist}\left(P_{1}, d\right)$. Thus we may choose $k_{1}$ as the smaller of the two numbers $m / 4 r$ and $m / \operatorname{diam}(D)$.

For the existence of $k_{2}$, let $M$ be an upper bound, estimated by (5.1), of $\Omega(S, P)$ for $\overline{S P} \geqq \delta / 3$. If dist $(P, d) \geqq \delta / 3$, then the inequality of the lemma is satisfied with $k_{2}=3 M / \delta$. Suppose that $P=P_{1}$ is a point of $D$ such that $\operatorname{dist}\left(P_{1}, d\right)<\delta / 3$. Let $S_{1}$ be a closest point of $d$ to $P_{1}$. If now $\overline{S_{1} P} \leqq \delta / 3$ and $\overline{S P}_{1} \geqq \delta$, then we have $\overline{S P} \geqq \delta / 3$, and hence $\Omega(S, P) \leqq M$. Let $\Sigma_{1}^{\prime}$ be the sphere of radius $r$ and center $Q_{1}^{\prime}$ externally tangent to $d$ at $S_{1}$; let $Q_{1}^{\prime \prime}$ be the midpoint of the segment $S_{1} Q_{1}^{\prime}$. The function $\phi\left(S_{1} ; P\right)=1-\left(\overline{S_{1} Q_{1}^{\prime \prime}} / \overline{P Q_{1}^{\prime \prime}}\right)$ is a barrier $\left.{ }^{27}\right)$ for $D$ at $S_{1}$. The greatest lower bound $m^{\prime}$ of $\phi\left(S_{1} ; P\right)$ for $\overline{P Q}_{1}^{\prime} \geqq r$, $\overline{S_{1} P}=\delta / 3$, is positive and depends only upon $r$ and $\delta$. From the properties of a barrier and the relations $\overline{P_{1} S_{1}}<\delta / 3$ and $\overline{P_{1} Q_{1}^{\prime \prime}}=\overline{P_{1} S_{1}}+\overline{S_{1} Q_{1}^{\prime \prime}}$ there follows for $\overline{S P_{1}} \geqq \delta, \Omega\left(S, P_{1}\right) \leqq\left(2 M / m^{\prime} r\right) \cdot \overline{S_{1} P_{1}}$. We now take $k_{2}$ as the larger of the two numbers $2 M / m^{\prime} r$ and $3 M / \delta$.

Assume now that a domain $D$ is the sum of two, $D_{1}$ and $D_{2}$, of which the latter is limited by a surface of bounded curvature. Consider the set $\sigma$ of boundary points of $D_{2}$ at a positive distance from $D_{1}$ and at a distance exceeding the positive number $\delta$ from that part $\tau$ of the boundary of $D_{1}$ lying in $D_{2}$. We assume that $\sigma$ is not void; thus, since it is open in the boundary of $D_{2}$, it has positive superficial measure. If now a positive mass lying in $D_{1}$ is swept out of $D$, the result is equivalent to that of commencing with $D_{1}$ and sweeping out $D_{1}$ and $D_{2}$ alternately in infinite succession. We seek a bound for the superficial density of the final distribution in points of $\sigma$.

LEMMA 2. Under the circumstances just mentioned, the distribution resulting from sweeping out $D$ has in each point of $\sigma$ a superficial density which varies continuously with the point and which nowhere in $\sigma$ exceeds $k \mu / A$, where $\mu$ is the total mass lying on $\tau$ after the first sweeping-out of $D_{1}$, where $A$ is the superficial measure of $\sigma$, and where $k$ is a constant which depends only upon the domain $D_{2}$ and the number $\delta$.

Let $f(S)$ be a function of the form

$$
f(S)=\int_{\tau} \Omega_{2}(S, P) d \mu\left(e_{P}\right),
$$

where $\mu(e)$ is a distribution of positive mass over $\tau$ and $\Omega_{2}(S, P)$ is $\Omega(S, P)$ for the domain $D_{2} . f(S)$ is a continuous function of $S$ in $\sigma$, and represents the density in the point $S$ of the distribution which results if we sweep the distribution $\mu(e)$ out of $D_{2}$. Now let $k=k_{2} / k_{1}$, where $k_{1}$ and $k_{2}$ are from Lemma 1 for $D_{2}$ and the present $\delta$. It follows readily from Lemma 1 and (5.2) that

(27) Kellogg, loc. cit., p. 329. 
$f(S) \leqq k \cdot f\left(S^{\prime}\right)$ for any two points $S$ and $S^{\prime}$ of $\sigma$. Thus, if we denote by $\bar{f}$ and $f$ respectively the least upper and greatest lower bounds of $f(S)$ in $\sigma$, we have $\bar{f} \leqq k \cdot f$.

Now let $f_{n}(S)$ be the density of the contribution to the mass on $\sigma$ made by the $n$th sweeping-out of $D_{2}$, and let $g_{n}(S)$ be the density of the total distribution on $\sigma$ at this stage. Since the total mass on $\sigma$ at no stage exceeds $\mu$, we have

$$
\sum_{1}^{n} f_{\nu}(S) \leqq \sum_{1}^{n} \bar{f}_{\nu} \leqq \sum_{1}^{n} k \cdot \underline{f}_{\nu} \leqq k \cdot \underline{g}_{n}=\frac{k}{A} \cdot \int_{\sigma} g_{n} d S \leqq \frac{k}{A} \cdot \int_{\sigma} g_{n}(S) \cdot d S \leqq \frac{k \mu}{A} \text {. }
$$

This relation not only shows that the infinite sum of the $f_{n}(S)$ representing the density of the final distribution is convergent and admits the desired upper bound, but also, since the sum of the $\bar{f}_{n}$ admits the same bound, that the convergence is uniform in $\sigma$.

Example 1. Domain with singular edge. Let $x, y, z$ be rectangular cartesian coordinates in space. We denote by $C(r)$, where $r>0$, the capsule-formed domain described by the conditions

$$
x^{2}+y^{2}<r^{2} ; \quad|z|<1+\left(r^{2}-x^{2}-y^{2}\right)^{1 / 2} .
$$

The surface bounding $C(r)$ evidently has bounded curvature. The segment of the $z$ axis for which $|z| \leqq 1$ we shall refer to as the core of $C(r)$. For $\alpha>0$, we shall denote by $C(r, \alpha)$ the configuration obtained from $C(r)$ by a translation $\alpha$ units in the direction of the positive $x$ axis.

Consider for fixed $r, r^{\prime}$ the intersection of the closures of the domains $C(r)$ and $C\left(r^{\prime}, \alpha\right)$. If $\alpha<r+r^{\prime}$, this is non-void, and, when $\alpha \uparrow r+r^{\prime}$, it closes down upon a line segment. Thus it is possible to choose $\alpha<\dot{r}+r^{\prime}$ so that the capacity of the intersection is arbitrarily small.

Now let $r_{0}, r_{1}, r_{2}, \cdots$ and $h_{1}, h_{2}, \cdots$ be respectively decreasing and increasing sequences of positive numbers of fixed selection subject only to the requirement that $r_{0}=1$, that the sum of the $r_{n}$ be convergent, and that $h_{n} \rightarrow \infty$. In terms of these sequences we define $(n=1,2, \cdots)$ :

$m_{n}$ as the minimum value of the function $\Omega(S, P)$ for the domain $C\left(r_{n}\right)$ when $S$ ranges over its boundary and $P$ over its core,

$A_{n}$ as the area of the surface bounding $C\left(r_{n}\right)$,

$k_{n}$ as the $k$ of Lemma 2 with $C\left(r_{n}\right)$ for $D_{2}$ and $\frac{1}{2} r_{n}$ for $\delta$,

$\alpha_{n}$ as a positive number chosen so that $r_{n-1}+\frac{1}{2} r_{n}<\alpha_{n}<r_{n-1}+r_{n}$ and so that the capacity of the intersection of the closures of $C\left(r_{n-1}\right)$ and $C\left(r_{n}, \alpha_{n}\right)$ is less than $m_{n} A_{n} / 2 k_{n} h_{n}$,

$$
\begin{aligned}
& \beta_{n} \text { as } \alpha_{1}+\alpha_{2}+\cdots+\alpha_{n}, \\
& C_{0} \text { as } C\left(r_{0}\right)=C(1), \text { and } C_{n} \text { as } C\left(r_{n}, \beta_{n}\right), \\
& D_{0} \text { as } C_{0}, \text { and } D_{n} \text { as } C_{0}+C_{1}+\cdots+C_{n} .
\end{aligned}
$$

The domains $C_{0}, C_{1}, C_{2}, \cdots$ are decreasing in size and lie parallel to each other with their centers in order along the positive $x$ axis. Two successive do- 
mains intersect each other, but neither contains the center of the other. The centers have as limit point the point $(\beta, 0,0)$, where $\beta=\lim \beta_{n}<2 \sum r_{n}$.

We now define the domain $D$ as $\lim D_{n} . D$ is bounded, and together with its boundary it is the topological image of a closed sphere. Every boundary point is regular with respect to the Dirichlet problem; in fact, Poincaré's "cone condition" applies in every boundary point. The boundary consists of parts of the boundaries of the $C_{n}$ and also the limiting line segment $x=\beta$, $y=0,|z| \leqq 1$. This segment we shall refer to as the singular edge of $D$. It is convenient to take for $P_{0}$ the center of $C_{0}$, that is, the origin of coordinates.

Theorem I. Any function $u(P)$ positive harmonic in $D_{n}$ taking on continuous boundary values which are zero for those boundary points not in $C_{n+1}$ satisfies

$$
u(P) \geqq h_{n} \cdot u\left(P_{0}\right)
$$

for all $P$ on the core of $C_{n}$. Thus, if $u(P)$ is positive harmonic in $D$ and approaches zero at every boundary point not on the singular edge, it is unbounded in the neighborhood of every point of the singular edge $\left({ }^{28}\right)$.

Consider $D_{n}$ as $D_{n-1}+C_{n}(n \geqq 1)$. Consider the set $\sigma_{n}$ of boundary points of $C_{n}$ whose distance from that part $\tau_{n}$ of the boundary of $D_{n-1}^{\prime}$ in $C_{n}$ exceeds $\frac{1}{2} r_{n}$. Since $\alpha_{n}>r_{n-1}+\frac{1}{2} r_{n}$, the set $\sigma_{n}$ includes all boundary points of $C_{n}$ whose $x$-coordinate exceeds $\beta_{n}$; thus, it has a superficial measure of at least $\frac{1}{2} A_{n}$. Since the distance of $\tau_{n}$ from $P_{0}$ is at least unity, the total mass received by $\tau_{n}$ when a unit mass at $P_{0}$ is swept out of $D_{n-1}$ cannot exceed the capacity of $\tau_{n}$, thus cannot exceed $m_{n} A_{n} / 2 h_{n} k_{n}$. It follows from Lemma 2 that the density in points of $\sigma_{n}$ of the distribution resulting when a unit mass at $P_{0}$ is swept out of $D_{n}$ cannot exceed $m_{n} / h_{n}$. On the other hand, when a unit mass at $P$ on the core of $C_{n}$ is swept out of $C_{n}$, the resulting density at points of $\sigma_{n}$ is at least $m_{n}$; this remains true $a$ fortiori if the sweeping-out is continued into $D_{n}$. Since the boundary points of $D_{n}$ in $C_{n+1}$ are in $\sigma_{n}$, the relation (5.3) is immediate from the estimates just made if $u(P)$ is represented in $D_{n}$ in terms of its boundary values by means of (1.1). The last statement of the theorem is an obvious corollary.

(28) This example, here introduced as auxiliary to the construction of Example 2, is not without intrinsic interest. First, it exhibits another way, besides that already pointed out by Bouligand, in which the principle of Picard may be in default. For, consider a point $Q$ of the singular edge. Instead of having at least two linearly independent positive harmonic functions approaching zero at all boundary points except $Q$, we have none at all. Second, with a suitable choice of the constants, the example serves to answer in the negative a conjecture of $N$. Wiener, Discontinuous boundary conditions and the Dirichlet problem, these Transactions, vol. 25 (1923), p. 313. For, if the numbers $h_{n}$ are so chosen that, say, $h_{n} / 2^{n} \rightarrow \infty$, then it is possible to define a non-negative, summable boundary function $\phi(Q)$ which vanishes at all boundary points $Q$ whose $z$-coordinate is less than unity, and which determines a harmonic function unbounded near all points of the singular edge. Thus, in particular, we have an example of a simple domain for which the condition $(\gamma)$, Maria and Martin, loc. cit., p. 519, is not fulfilled. 
Example 2. Domain for which $\Delta_{0}$ is non-void. We write $D_{2}$ for the $D$ of Example 1. Retaining the notations introduced in connection with that example, let $A$ and $A^{\prime}$ be the end-points of the singular edge, and let $\Sigma$ and $\Sigma^{\prime}$ be open spheres of radius $\frac{1}{2}(<1)$ having these points respectively for centers $\left({ }^{29}\right)$. We define the domain $D$ as $D_{2}+\Sigma+\Sigma^{\prime}$. This domain is symmetric about the plane $z=0$, it has the same simple connectivity as does $D_{2}$, and it is regular with respect to the Dirichlet problem. It is convenient also to define the auxiliary domain $D_{1}=D_{2}-\bar{\Sigma}-\bar{\Sigma}^{\prime}$. Since $D_{1} \subset D_{2} \subset D$, we have

$$
G_{1}(M, P)<G_{2}(M, P)<G(M, P)
$$

for the corresponding Green's functions.

Form the functions $K_{2}(M, P)$ and $K(M, P)$ corresponding respectively to $D_{2}$ and $D$ as in $\S 2$, taking in both cases $P_{0}$ as the origin of coordinates. Consider a sequence $\left\{M_{n}\right\}$ of points which lie on the $x$ axis and whose $x$ coordinates form an increasing sequence with limit $\beta$. Without loss of generality it may be assumed that $\left\{M_{n}\right\}$ is fundamental for both $D_{2}$ and $D$, thus determining for them ideal boundary elements $S_{2}$ and $S$ together with the corresponding harmonic functions $K_{2}\left(S_{2}, P\right)$ and $K(S, P)$. We shall prove that $K(S, P)$ is not minimal for $D$.

In $D_{1}$ we have

$$
G(M, P)=G_{1}(M, P)+G_{\left[\Sigma+\Sigma^{\prime}\right]}^{*}(M, P) .
$$

From this, on writing $v_{n}(P)=K_{\left[\Sigma+\Sigma^{\prime}\right]}^{*}\left(M_{n}, P\right)$ and $w_{n}(P)=G_{1}\left(M_{n}, P\right) / G\left(M_{n}, P_{0}\right)$,

$$
K\left(M_{n}, P\right)=w_{n}(P)+v_{n}(P) .
$$

Now $w_{n}(P) \rightarrow 0$ in $D_{1}$. If not, a subsequence would have a positive harmonic limit $w(P)$ in $D_{1}$. If $k$ were any positive number less than $w\left(P_{0}\right)$, we should have infinitely many $n$ such that $w_{n}\left(P_{0}\right)=G_{1}\left(M_{n}, P_{0}\right) / G\left(M_{n}, P_{0}\right)>k$. This would imply that

$$
\begin{aligned}
K_{2}\left(S_{2}, P\right) & =\lim _{n \rightarrow \infty} \frac{G_{2}\left(M_{n}, P\right)}{G_{2}\left(M_{n}, P_{0}\right)} \leqq \liminf _{n \rightarrow \infty} \frac{G\left(M_{n}, P\right)}{G_{1}\left(M_{n}, P_{0}\right)} \\
& \leqq \limsup _{n \rightarrow \infty} \frac{1}{k} \cdot \frac{G\left(M_{n}, P\right)}{G\left(M_{n}, P_{0}\right)}=\frac{1}{k} \cdot K(S, P) .
\end{aligned}
$$

But this is impossible since, in particular, $K(S, P)$ is harmonic at $P=A$, while $K_{2}\left(S_{2}, P\right)$, as follows readily from Theorem $\mathrm{I}$, is unbounded near $A$. Thus we have proved that $v_{n}(P) \rightarrow K(S, P)$ for $P$ in $D_{1}$.

The functions $v_{n}(P)$ approach zero in every boundary point of $D$; further-

(29) The device of forming two modified domains by means of a sphere (here two spheres) is due to A. J. Maria. The argument below which enables us to prove the boundedness of $K(S, P)$ at points distant from $\Sigma+\Sigma^{\prime}$ is a paraphrase of that shown the author by Maria some time ago. 
more, they are uniformly bounded over any set at a positive distance from $\Sigma+\Sigma^{\prime}$. In fact,

$$
v_{n}(P) \leqq K_{[\Sigma]}^{*}\left(M_{n}, P\right)+K_{\left[\Sigma^{\prime}\right]}^{*}\left(M_{n}, P\right),
$$

and, for $\overline{A P}>\frac{1}{2}$, we have $\left.{ }^{30}\right)$

$$
K_{[\Sigma]}^{*}\left(M_{n}, P\right) \leqq K\left(M_{n}, A\right) \cdot \frac{2 \cdot \bar{A} \bar{P}+1}{(2 \cdot \bar{A} \bar{P}-1)^{2}},
$$

together with an analogous bound for the other function when $\overline{A^{\prime} P}>\frac{1}{2}$. This result, coupled with the result of the preceding paragraph, implies that $K(S, P)$ approaches zero in every boundary point of $D$ which is at a positive distance from the spheres $\Sigma$ and $\Sigma^{\prime}$.

Now let $\sigma$ be the closure of the set of those boundary points of $D$ which are limit points of $\Sigma$ and for which $K(S, P)$ has a positive superior limit $\left({ }^{31}\right)$. Similarly define $\sigma^{\prime}$ in terms of $\Sigma^{\prime}$. Since $K(S, P)$ is symmetrical about the $x y$ plane, $\sigma$ and $\sigma^{\prime}$ are reflections of each other in it. Let $\sigma_{n}$ and $\sigma_{n}^{\prime}$ be the sets of points of $D$ whose distance from $\sigma$ and $\sigma^{\prime}$ respectively does not exceed $1 / n$. In $D-\sigma_{n}-\sigma_{n}^{\prime}, K(S, P)$ is bounded and takes on continuous boundary values. These are zero at those boundary points which are also boundary points of $D$. Thus,

$$
K(S, P)=K_{\left(\sigma_{n}+\sigma_{n}\right)}^{*}(S, P) .
$$

Write $u_{n}(P)=K_{\sigma_{n}}^{*}(S, P)$. If $n>1$, the boundary values determining this function in $D-\sigma_{n}$ are continuous; hence the function itself must approach zero in every boundary point of $D$ not in $\sigma$. Since as $n \rightarrow \infty$ the $u_{n}(P)$ form a descending sequence, they have a limit $u(P)$ which is non-negative harmonic in $D$ and which approaches zero in every boundary point not in $\sigma$. An analogous statement holds for the functions $u_{n}^{\prime}(P)$ similarly defined in terms of $\sigma_{n}^{\prime}$ and for their limit $u^{\prime}(P)$. By the symmetry of the construction, the functions $u(P)$ and $u^{\prime}(P)$ are images of each other in the $x y$ plane. Neither function can be zero, since (5.4) in conjunction with (h) of Theorem II, $\S 1$, shows that $K(S, P)$ is dominated by $u_{n}(P)+u_{n}^{\prime}(P)$ and hence by $u(P)+u^{\prime}(P)$. Now $K(S, P)$ dominates both $u(P)$ and $u^{\prime}(P)$. Thus, if $K(S, P)$ were minimal, these functions would be multiples of each other. This would give a contradiction, since, by what was shown above, it would imply that both functions approach zero in every boundary point of $D$. This proves

THEOREM II. There exist structurally simple domains for which the set $\Delta_{0}$ is not vacuous.

$\left.{ }^{(3 \theta}\right)$ This is readily shown by considering a smaller sphere $\Sigma_{1}$ concentric with $\Sigma$, solving the exterior Dirichlet problem for $\Sigma_{1}$ with boundary values $\phi(Q)=K\left(M_{n}, Q\right)$, and making $\Sigma_{1} \uparrow \Sigma$.

( $\left.{ }^{31}\right)$ The set $\sigma$ actually consists of the single point in which the surface of $\Sigma$ intersects the singular edge of $D_{2}$. 
Let us turn now to another point. The definition of ideal boundary point in $\$ 2$ is purely potential-theoretic in the sense that it involves the actual structure of the domain only indirectly as the structure influences the behavior of Green's functions. It is natural to ask whether these boundary elements are identifiable with some suitable topologically defined boundary elements analogous, perhaps, to prime-ends $\left({ }^{32}\right)$. We show by Example 3 that if by this there is meant a purely topological definition, that is, naturally, one which is invariant under topological mappings of two domains together with their boundaries upon each other, then the answer is in the negative. This, of course, does not preclude the possibility of obtaining a more geometric definition equivalent to that of $\S 2$, but indicates, rather, that such a definition must take into account the metric structure of the domain as well. The domain is of sufficient simplicity to permit the carrying out explicitly of the representation of the positive harmonic functions in the terms of the present analysis, and we do so.

Example 3. A simple domain in which a certain boundary point corresponds to a continuum of ideal boundary elements. The domain we consider is one of the two bounded by the surface of two spheres, one internally tangent to the other, and by a plane containing the common diameter. The boundary point of interest is naturally the point of tangency of the bounding spheres. By a succession of inversions commencing with an inversion about this point the domain can be thrown into $\left({ }^{33}\right)$

$$
D: \quad 0<x<\infty, \quad-\infty<y<\infty, \quad-\frac{1}{2} \pi<z<\frac{1}{2} \pi .
$$

The corresponding Kelvin transformation reduces the study of the positive harmonic functions of the original domain to the study of those of $D$. Evidently the minimal property is preserved under the transformation.

For this discussion we shall designate $(\xi, \eta, \zeta)$ as the coordinates of a point $M$ of $D$ and by $(x, y, z)$ those of $P .(\rho, \phi, \zeta)$ and $(r, \theta, z)$, where $\xi=\rho \cos \phi$, etc., will be the corresponding cylindrical coordinates. For $P_{0}$ we choose $(1,0,0)$.

By the methods of images, the Green's function $G(M, P)$ for $D$ may be determined as a conditionally convergent lattice potential:

$$
G(M, P)=\sum_{\mu=-\infty}^{\infty} \sum_{\nu=0}^{1}(-1)^{\mu+\nu} \overline{(M)_{\mu, \nu}}-1,
$$

in which $(M)_{\mu, \nu}$ denotes the point $\left[(-1)^{\nu} \xi, \eta, \mu \pi+(-1)^{\mu} \zeta\right]$.

(32) In particular, we may cite the definition of boundary element given by F. W. Perkins, The Dirichlet problem for domains with multiple boundary points, these Transactions, vol. 38 (1935), pp. 106-144. In so far as the results of Green, loc. cit., are dependent upon this definition of boundary element, there will necessarily be a point of divergence from the present analysis.

$\left.{ }^{(33}\right)$ Exactly this domain $D$ has been considered from a closely allied viewpoint of Bouligand, loc. cit. (Étude des singularités, . . ), pp. 140-144. Certain of the facts here derived are contained in his work. 
We form the function $K(M, P)$ and suppose that $M$ runs through a sequence having no point of accumulation in $D$. Two cases are to be distinguished: (1) $M \rightarrow S$, where $S$ is a boundary point of $D$; (2) $\rho \rightarrow \infty$ and $\phi \rightarrow \alpha$ where $\alpha$ is some angle between $-\frac{1}{2} \pi$ and $\frac{1}{2} \pi$ inclusive.

In the first case, $K(M, P)$ approaches a harmonic limit in $D$ which depends upon $S$ but not on the particular manner of approach. The limit function approaches zero as $P$ approaches any boundary point of $D$ distinct from $S$ and also approaches zero at infinity. To see this, we may observe that the series in (5.5) extends the definition of $G(M, P)$ to the whole of space. In particular, when $M$ does not coincide with $P$ or any of its image points, $G(M, P)$ is analytic in $\xi, \eta$, and $\zeta$, of period $2 \pi$ in $\zeta$, odd in $\zeta \pm \frac{1}{2} \pi$, and odd in $\xi$; similar statements hold in $x, y$, and $z$. From this it follows that if we write $h(M)=\xi \cos \zeta$, the quotient $G(M, P) / h(M)$ has a determinate limit $H(S, P)$ as $M \rightarrow S$. Writing $\left(\xi_{0}, \eta_{0}, \zeta_{0}\right)$ for the coordinates of $S$, we have

$$
H(S, P)= \begin{cases}\left(\frac{\partial G}{\partial \xi} / \frac{\partial h}{\partial \xi}\right)_{M=S}\left(\xi_{0}=0 ; \zeta_{0} \neq \pm \frac{1}{2} \pi\right) & \\ \left(\frac{\partial G}{\partial \zeta} / \frac{\partial h}{\partial \zeta}\right)_{M=S} & \left(\xi_{0} \neq 0 ; \zeta_{0}= \pm \frac{1}{2} \pi\right) \\ \left(\frac{\partial^{2} G}{\partial \xi \partial \zeta} / \frac{\partial^{2} h}{\partial \xi \partial \zeta}\right)_{M=S} & \left(\xi_{0}=0 ; \zeta_{0}= \pm \frac{1}{2} \pi\right) .\end{cases}
$$

A straightforward computation of these derivatives in (5.5) shows that $H(S, P)$, as a function of $P$, has singularities at $S$ and its image points and only there; furthermore that it approaches zero as $P$ moves to infinity in $D$. Because of the presence of these singularities, $H(S, P)$ cannot vanish identically. In particular, $H(S, P)$ must be positive harmonic in $D$. Since the parity relations of $G(M, P)$ in $x$ and $z$ are preserved in $H(S, P)$, the latter function must vanish in every boundary point of $D$ distinct from $S$. Finally,

$$
\frac{H(S, P)}{H\left(S, P_{0}\right)}=\lim _{M \rightarrow S} \frac{G(M, P) / h(M)}{G\left(M, P_{0}\right) / h(M)}=\lim _{M \rightarrow S} K(M, P) .
$$

In the second case, $K(M, P)$ approaches a positive harmonic limit which depends upon $\alpha$ but is otherwise independent of the manner of approach of $M$ to infinity. The limit function, which approaches zero in every boundary point of $D$ but is unbounded at infinity, is given explicitly by

$$
\begin{array}{cc}
\operatorname{csch}(\cos \alpha) \cdot \cos z \cdot \exp (y \sin \alpha) \cdot \sinh (x \cos \alpha) & \left(|\alpha|<\frac{1}{2} \pi\right), \\
x \cdot \cos z \cdot \exp (y \sin \alpha) & \left(\alpha= \pm \frac{1}{2} \pi\right) .
\end{array}
$$

This result can be derived from an asymptotic development for $G(M, P)$ valid as $M$ recedes to infinity. To obtain such an expression from (5.5) we apply contour integration to each of the functions 


$$
\cos \zeta[\sin \zeta-\sin t]^{-1}\left[(x \pm \xi)^{2}+(y-\eta)^{2}+(z-t)^{2}\right]^{-1 / 2}
$$

made single valued in the complex $t$ plane by cuts parallel to the imaginary axis from the branch points to infinity, the integration in each case being around the boundary of the cut plane. The result is

$$
G(M, P)=\int_{0}^{\infty}\left\{\frac{\phi(\zeta, z ; \lambda+p)}{\left(\lambda^{2}+2 \lambda p\right)^{1 / 2}}-\frac{\phi(\zeta, z ; \lambda+q)}{\left(\lambda^{2}+2 \lambda q\right)^{1 / 2}}\right\} d \lambda,
$$

where

$$
\begin{gathered}
p=\left[(x-\xi)^{2}+(y-\eta)^{2}\right]^{1 / 2}, \quad q=\left[(x+\xi)^{2}+(y-\eta)^{2}\right]^{1 / 2}, \\
\phi(\zeta, z ; \lambda)=\frac{2}{\pi} \cdot \frac{\cos \zeta \cdot \cos z \cdot \sinh \lambda}{[\cosh \lambda+\cos (\zeta+z)][\cosh \lambda-\cos (\zeta-z)]} .
\end{gathered}
$$

Now evidently as $\lambda \rightarrow \infty$ we have, uniformly in $\zeta$ and $z, \phi(\zeta, z ; \lambda) \sim(4 / \pi) \cos \zeta$ $\cdot \cos z \cdot e^{-\lambda}$, where by this is meant that the quotient of the two functions approaches unity. Also, as $\rho \rightarrow \infty$, uniformly for $r$ bounded and $\lambda>0$,

$$
\begin{gathered}
p=\rho-r \cos (\theta-\phi)+O\left(\rho^{-1}\right) ; q=\rho+r \cos (\theta+\phi)+O\left(\rho^{-1}\right) ; \\
\left(\lambda^{2}+2 \lambda p\right)^{1 / 2} \sim\left(\lambda^{2}+2 \lambda q\right)^{1 / 2} \sim\left(\lambda^{2}+2 \lambda \rho\right)^{1 / 2} .
\end{gathered}
$$

From these and (5.7) we have as $\rho \rightarrow \infty$, uniformly for $r$ bounded,

$$
G(M, P) \sim \cos \zeta \cdot \cos z \cdot\{\exp [r \cos (\theta-\phi)]-\exp [-r \cos (\theta-\phi)]\} \cdot \Phi(\rho),
$$

in which

$$
\Phi(\rho)=\frac{4}{\pi} \int_{0}^{\infty} \frac{e^{-(\lambda+\rho)}}{\left(\lambda^{2}+2 \lambda \rho\right)^{1 / 2}} d \lambda .
$$

The expressions in (5.6) are now immediate.

Since a sequence of points $M$ having no point of accumulation in $D$ has a subsequence falling under one or the other of the above two cases, it follows from the results of the preceding two paragraphs that these cases subsume all fundamental sequences. Thus the ideal boundary elements of $D$ fall into two corresponding classes. Those of the first class may be identified with boundary points of $D$, the relative neighborhoods in $D$ being the same as those of the corresponding boundary point. The same symbol will be used to designate a boundary point of $D$ and its corresponding boundary element of this class. The ideal boundary elements of the second class are to be identified with directions $\alpha$ of approach to infinity. We denote these elements by $S_{\alpha}$, where $-\frac{1}{2} \pi \leqq \alpha \leqq \frac{1}{2} \pi$. The sets $N(R, \beta, \gamma)$ consisting of all $P$ in $D$ for which $r>R$ and $\beta<\theta<\gamma$, where $R \geqq 0$ and $-\frac{1}{2} \pi \leqq \beta<\gamma \leqq \frac{1}{2} \pi$ form a convenient system of relative neighborhoods in $D$ of the boundary elements $S_{\alpha}$.

It is now readily proved that the set $\Delta_{0}$ for $D$ is void. First, let $S$ be a boundary element of the first class, and let $\sigma$ be the closure in $D$ of a relative 
neighborhood of $S$. By an argument similar to that used to establish (5.4), we obtain $K_{\sigma}^{*}(S, P)=K(S, P)$; from this follows $\psi(S)=1$. To prove that the functions $K\left(S_{\alpha}, P\right)$ are minimal, assume first that $-\frac{1}{2} \pi<\alpha<\frac{1}{2} \pi$. Let $\sigma(R, \beta, \gamma)$ be the closure in $D$ of the set $N(R, \beta, \gamma)$ above. It is sufficient to show that $F(\alpha, R, \beta, \gamma) \equiv K_{\sigma(R, \beta, \gamma)}^{*}\left(S_{\alpha}, P_{0}\right)=1$ whenever $\beta<\alpha<\gamma$. That the latter is true when $\beta=-\frac{1}{2} \pi$ and $\gamma=\frac{1}{2} \pi$ is easily shown by another argument similar to that cited above from (5.4). Using (h), Theorem II, $\$ 1$, we then have

$$
\begin{aligned}
F\left(\alpha, R,-\frac{1}{2} \pi, \beta\right)+F(\alpha, R, \beta, \gamma)+F(\alpha, R, \gamma, & \left.\frac{1}{2} \pi\right) \\
& \geqq F\left(\alpha, R,-\frac{1}{2} \pi, \frac{1}{2} \pi\right)=1 .
\end{aligned}
$$

Since $F$ is a positive non-increasing function of $R$ whose value never exceeds unity, it suffices to show that the first and third terms of the first member of (5.8) approach zero as $R \rightarrow \infty$. Consider, for example, $F\left(\alpha, R,-\frac{1}{2} \pi, \beta\right)$. Let $M(R)$ be the maximum value of the quotient $K\left(S_{\alpha}, P\right) / K\left(S_{\beta}, P\right)$ for $P$ in $\sigma=\sigma\left(R,-\frac{1}{2} \pi, \beta\right)$. By a direct estimate from (5.6) we have $M(R)=O(\exp$ $[R \cdot \cos (\alpha-\beta)-R]) \rightarrow 0$ as $R \rightarrow \infty$. Also $F\left(\alpha, R,-\frac{1}{2} \pi, \beta\right)=K_{\sigma}^{*}\left(S_{\alpha}, P_{0}\right) \leqq M(R)$ $\cdot K_{\sigma}^{*}\left(S_{\beta}, P_{0}\right) \leqq M(R)$. This completes the proof for $-\frac{1}{2} \pi<\alpha<\frac{1}{2} \pi$. If $\alpha$ has one of the extreme values, say $\frac{1}{2} \pi$, we write $\gamma=\frac{1}{2} \pi$, and omit the third term of the first member of (5.8). The rest of the proof proceeds as before.

Now return to the original domain bounded by the two spheres and the plane. Denote this domain by $D^{\prime}$, and by $T$ the point of tangency of the two bounding spheres. If $P_{0}^{\prime}$ is the image of $P_{0}$ under the conformal transformation which maps $D$ on $D^{\prime}$, then, as is readily verified, the functions $K^{\prime}(M, P)$ formed for $D^{\prime}$ with normalization at $P_{0}^{\prime}$ are fixed multiples of the Kelvin transforms of the functions $K(M, P)$ for $D$. Thus the neighborhoods in $D^{\prime}$ of its ideal boundary elements are the images under the mapping of the corresponding neighborhoods in $D$. In particular, neighborhoods in $D$ of boundary elements of the first class map into ordinary neighborhoods in $D^{\prime}$ of its boundary points distinct from $T$; the neighborhoods $N(R, \beta, \gamma)$ of the $S_{\alpha}$ map into fang-shaped sets in $D^{\prime}$ each having at its apex the point $T$. We denote by $T_{\alpha}$ the ideal boundary element in $D^{\prime}$ corresponding to $S_{\alpha}$ in $D$. The set of all $T_{\alpha}$ $\left(-\frac{1}{2} \pi \leqq \alpha \leqq \frac{1}{2} \pi\right)$ is evidently closed in the ideal boundary of $D^{\prime}$. The general representation of $\S 3$ coupled with the uniqueness theorem of $\$ 4$ now gives the following representation for the most general positive harmonic in $D^{\prime}$ :

$$
u(P)=\int_{d^{\prime}-\{T\}} K^{\prime}(Q, P) d \mu\left(e_{Q}\right)+\int_{-\pi / 2}^{\pi / 2} K^{\prime}\left(T_{\alpha}, P\right) d \nu\left(e_{\alpha}\right),
$$

where $\mu(e)$ is a non-negative distribution over $d^{\prime}-\{T\}$ and $\nu(e)$ is a similar distribution over the interval $\left(-\frac{1}{2} \pi, \frac{1}{2} \pi\right) . \mu(e)$ and $\nu(e)$ are both uniquely determined by $u(P)$.

The University of Illinois, URBana, Ill. 